\title{
Cyclic RGD-Decorated Liposomal Gossypol AT-I0I Targeting for Enhanced Antitumor Effect
}

\author{
Hao Liu, (D)' Ruirui Zhang, ' \\ Dan Zhang,' Chun Zhang, ' \\ Zhuo Zhang,' Xiujuan Fu,' \\ Yu Luo,' Siwei Chen,' \\ Ailing Wu, ${ }^{2}$ Weiling Zeng, ${ }^{3}$ \\ Kunyan Qu,' Hao Zhang,' \\ Sijiao Wang, ' Houyin Shi ${ }^{4}$ \\ 'School of Pharmacy, Southwest Medical \\ University, Luzhou City, Sichuan, People's \\ Republic of China; ${ }^{2}$ Department of \\ Anesthesiology, The First People's Hospital \\ of Neijiang, Neijiang, Sichuan, People's \\ Republic of China; ${ }^{3}$ Department of Scientific \\ Research, The Affiliated Traditional Chinese \\ Medicine Hospital of Southwest Medical \\ University, Luzhou City, Sichuan, People's \\ Republic of China; ${ }^{4}$ Department of \\ Orthopedics, The Affiliated Traditional \\ Chinese Medicine Hospital of Southwest \\ Medical University, Luzhou City, Sichuan, \\ People's Republic of China
}

Correspondence: Hao Liu School of Pharmacy, Southwest Medical University, No. I Section I, Xiang Lin Road, Longmatan District, Luzhou City, Sichuan Province, 646000, People's Republic of China

Tel +86830 3162291

Email h_lewis@I26.com

Houyin Shi

Department of Orthopedics, The Affiliated Traditional Chinese Medicine Hospital of Southwest Medical University, No. 182 Chunhui Road, Longmatan District, Luzhou City, Sichuan Province, 646000, People's Republic of China Tel +868303162209

Email623340767@qq.com
Introduction: (-)-Gossypol (AT-101), the (-)-enantiomer of the natural compound gossypol, has shown significant inhibitory effects on various types of cancers such as osteosarcoma, myeloma, glioma, lung cancer, and prostate cancer. However, the clinical application of (-)gossypol was often hindered by its evident side effects and the low bioavailability via oral administration, which necessitated the development of suitable (-)-gossypol preparations to settle the problems. In this study, injectable cyclic RGD (cRGD)-decorated liposome (cRGD-LP) was prepared for tumor-targeted delivery of (-)-gossypol.

Methods: The cRGD-LP was prepared based on cRGD-modified lipids. For comparison, a noncRGD-containing liposome (LP) with a similar chemical composition to cRGD-LP was specially designed. The physicochemical properties of (-)-gossypol-loaded cRGD-LP (Gos/cRGD-LP) were investigated in terms of the drug loading efficiency, particle size, morphology, drug release, and so on. The inhibitory effect of Gos/cRGD-LP on the proliferation of tumor cells in vitro was evaluated using different cell lines. The biodistribution of cRGD-LP in vivo was investigated via the near-infrared (NIR) fluorescence imaging technique. The antitumor effect of Gos/cRGD-LP in vivo was evaluated in PC-3 tumor-bearing nude mice.

Results: Gos/cRGD-LP had an average particle size of about $62 \mathrm{~nm}$ with a narrow size distribution, drug loading efficiency of over $90 \%$, and sustained drug release for over 96 h. The results of NIR fluorescence imaging demonstrated the enhanced tumor targeting of cRGD-LP in vivo. Moreover, Gos/cRGD-LP showed a significantly enhanced inhibitory effect on PC-3 tumors in mice, with a tumor inhibition rate of over $74 \%$ and good biocompatibility.

Conclusion: The incorporation of cRGD could significantly enhance the tumor-targeting effect of the liposomes and improve the antitumor effect of the liposomal (-)-gossypol in vivo, which indicated the potential of Gos/cRGD-LP that warrants further investigation for clinical applications of this single-isomer drug.

Keywords: RGD peptide, $\alpha_{\mathrm{v}} \beta_{3}$ integrin, targeted therapy, AT-101, liposome, fluorescence imaging

\section{Introduction}

Gossypol (2,2'-bis-(formyl-1,6,7-trihydroxy-5-isopropyl-3-methylnaphthalene) is a natural compound found in cotton plants. In the past few years, it was reported to show notable anticancer effects, along with antivirus, antiparasitic, and antioxidant properties. ${ }^{1,2}$ More recent studies found that it is the (-)-enantiomer of gossypol (Figure 1A), namely (-)-gossypol (also known as AT-101), rather than (+)-gossypol or the racemic gossypol that has significant anticancer properties. ${ }^{1,3}$ In this situation, it is necessary to develop single-isomer drug preparations to reduce the ineffective substance to patients and avoid potential adverse/side effects. ${ }^{4}$ Despite the various mechanisms 


\section{Graphical Abstract}

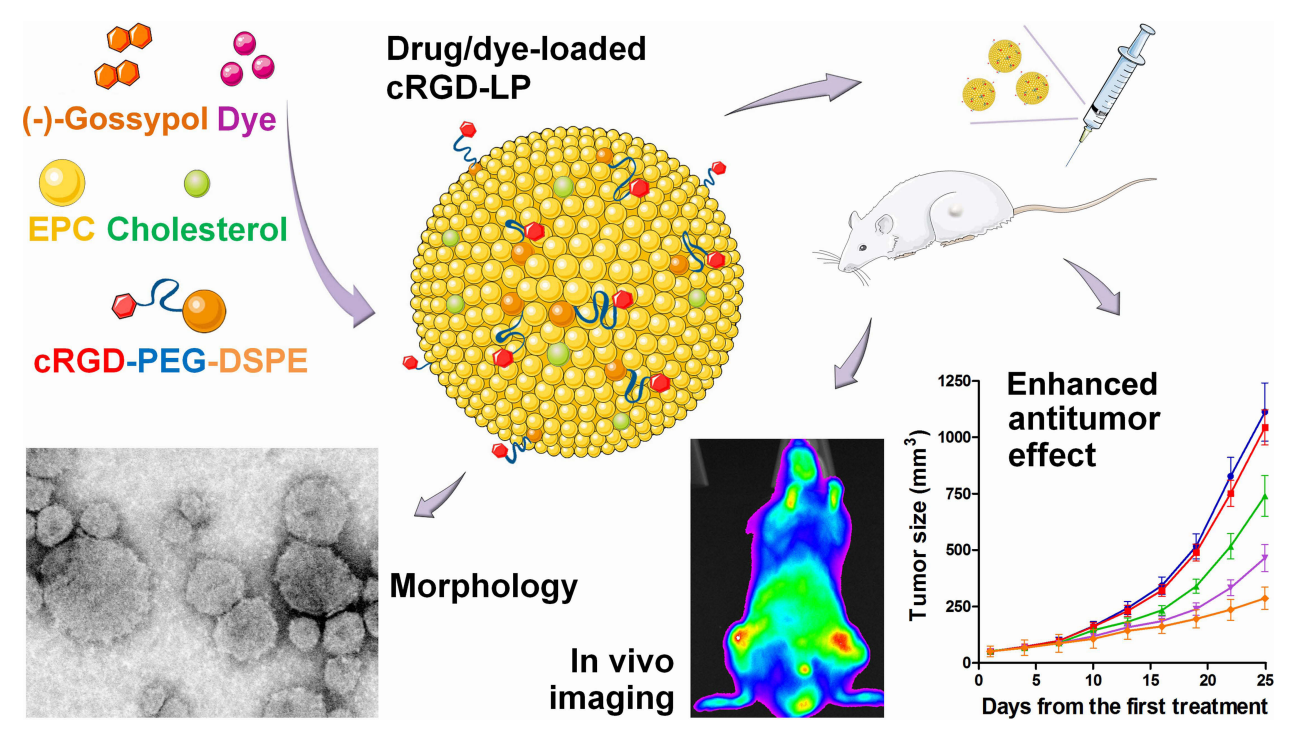

reported so far, the cancer cell suppression effect of (-)gossypol is mainly realized via either apoptotic cell death or autophagic cell death. ${ }^{3,5}$ For apoptosis-proficient cells, (-)gossypol may induce apoptotic cell death by caspasedependent pathway, mitochondrial pathway, and/or cell cycle arrest. ${ }^{5}$ For apoptosis-deficient cells, (-)-gossypol may trigger autophagic cell death by deterring Bcl-2-Beclin 1 interaction. So far, (-)-gossypol has been investigated as a promising anticancer drug for the therapy of various types of cancers/tumors such as osteosarcoma, ${ }^{6}$ myeloma, ${ }^{7}$ glioma,${ }^{8}$ lung cancer, ${ }^{9,10}$ prostate cancer, ${ }^{11-13}$ and adrenal cortical carcinoma. ${ }^{14}$ Although many previous studies of (-)-gossypol resulted in evident anticancer effects, the clinical application of this drug has been hindered by some shortcomings. Firstly, since (-)gossypol molecule is hydrophobic, it cannot be easily made into solutions with an adequate drug concentration for injection. For this reason, (-)-gossypol has to be administered orally in almost all clinical trials, which may have reduced the drug bioavailability and consequently compromised the therapeutic efficacy. ${ }^{10,13,14}$ As a result, combined therapy using (-)gossypol with other anticancer drugs was often needed for better therapeutic efficacy in clinical trials. ${ }^{15-19}$ Moreover, oral (-)-gossypol sometimes leads to gastrointestinal-related side effects such as stomach discomfort, anorexia, and nausea/ vomiting. ${ }^{10}$ Secondly, the biodistribution of (-)-gossypol is not specific to tumors, which may result in systemic toxicity or toxicity in the normal organs/tissues..$^{10,13,14}$ The above problems necessitated the development of strategies for tumortargeted delivery of (-)-gossypol to achieve improved therapeutic efficacy and minimize the adverse/side effects.

To date, there have been only a few tumor-targeted preparations for (-)-gossypol reported. Previously, (-)gossypol-loaded cubosomes and polymeric nanoparticles were investigated, respectively. ${ }^{20,21}$ Both of the two preparations showed effective tumor cell inhibition in vitro, but the drug efficacy in vivo was not clear. Tomoda et al reported an injectable (-)-gossypol-loaded Pluronic P85 micelle which had limited antitumor efficacy alone but could significantly enhance the antitumor efficacy of radiation therapy. ${ }^{22}$ Earlier, Li et al reported liposomes that encapsulated (-)-gossypol-enriched cottonseed oil. ${ }^{23}$ However, the cottonseed oil contained a mixture of (-)gossypol (65\%) and (+)-gossypol (35\%) rather than a single-isomer drug. In addition, for all the four preparations above, the potential passive tumor-targeting effect of the formulations in vivo was not clear. ${ }^{20-23}$ Therefore, there is still a lot of work to be done before a practicable tumor-targeted formulation for (-)-gossypol can be obtained. Recently, nanocarriers consisting of mixed materials have provided a strategy to achieve multiple goals at the same time. ${ }^{24,25}$ We previously reported a doublelayered (-)-gossypol-loaded nanoparticle [(-)-G-PgSHA] containing chemically modified polyethyleneimine and hyaluronic acid. ${ }^{26}$ The modified polyethyleneimine could 
A

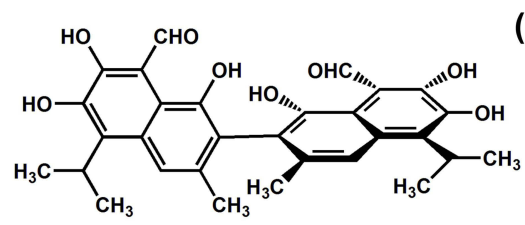

\section{(-)-Gossypol}

B

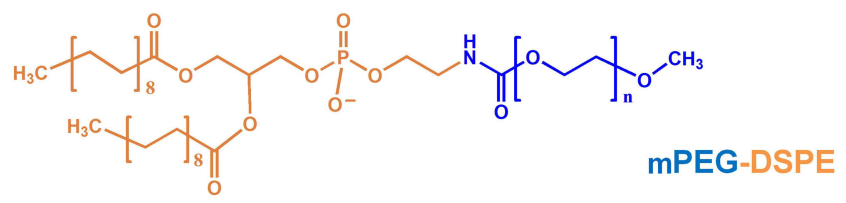

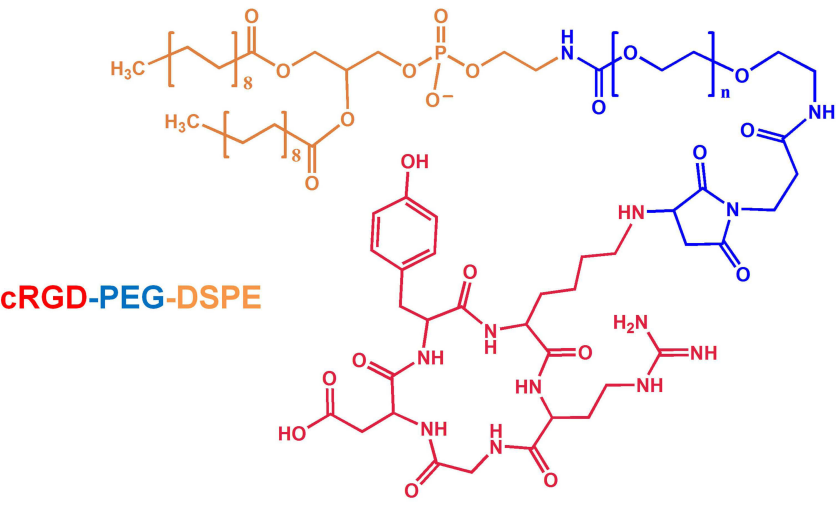

\section{C}

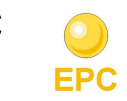

$$
\text { EPC }
$$

Cholesterol

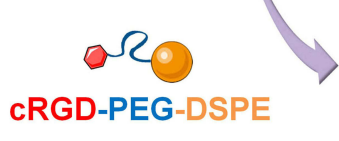

Thin-film hydration
ISonication

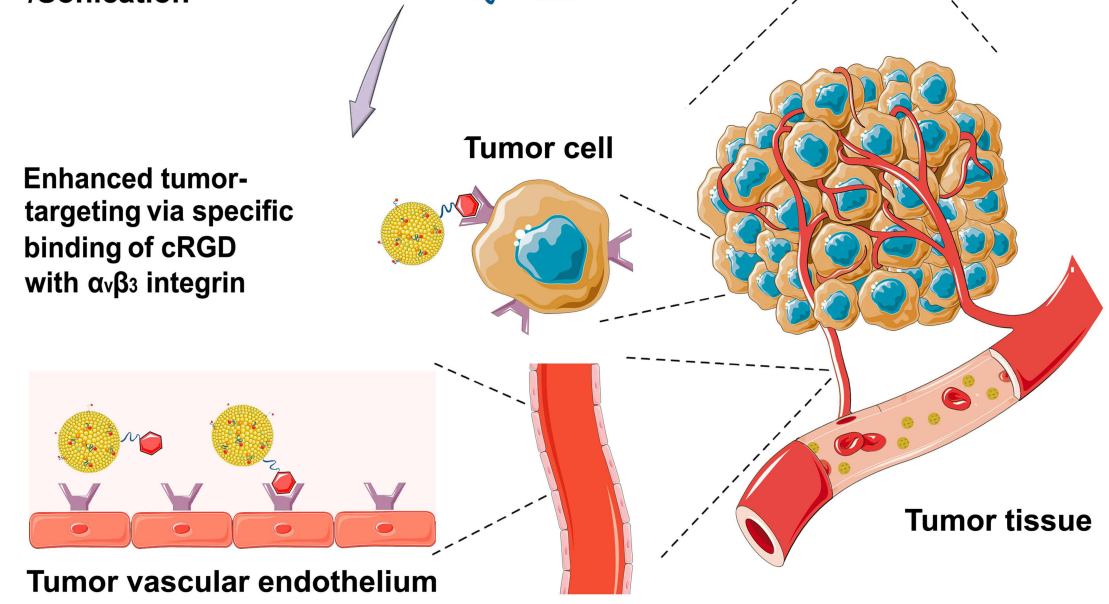

Figure I Schematic illustration for (A) the chemical structure of (-)-gossypol; (B) the chemical structures of mPEG-DSPE and cRGD-PEG-DSPE; (C) the design and preparation of cRGD-decorated liposome (cRGD-LP) with enhanced tumor-targeting for drug delivery. 
result in better stability and loading efficiency of (-)gossypol via its hydrogen bonding with the drug molecule. Hyaluronic acid on the surface of the drug-loaded nanoparticle could enhance the antitumor efficacy via CD44mediated endocytosis. Nevertheless, polyethyleneimine has shown significant cytotoxicity, which made it improper for practical use, especially for long-term administration. The stability of (-)-G-PgSHA was not satisfactory at room temperatures, which required strict conditions for storage. In addition, the preparation process for (-)-G-PgSHA was too time-consuming and complex for large-scale production. $^{26}$

In this study, cyclic RGD (cRGD)-decorated mixed liposome (cRGD-LP) for tumor-targeted delivery of (-)gossypol (abbreviated as Gos in this study) was prepared. The cRGD peptides with high affinity to $\alpha_{v} \beta_{3}$ integrin have been widely used for targeting tumor vasculature and/or tumor cells. ${ }^{27}$ Recently, Yang et al designed a composite nanoparticle (cRGD-PSH-NP) based on modified polyethyleneimine and cRGD-conjugated lipids for targeted delivery of survivin siRNA. ${ }^{28}$ The cRGD-PSHNP was low in toxicity and had a good loading ability for survivin siRNA. The survivin siRNA-loaded cRGD-PSHNP exhibited a tumor inhibition rate of up to about $75 \%$ in HepG2-bearing nude mice with excellent safety. Fu et al prepared cRGD-modified doxorubicin (DOX)-loaded liposomes and compared them with linear RGD-modified or non-modified liposomes. ${ }^{29}$ It turned out that the cRGDmodified liposomes had the most accumulation at the tumor sites in A549 tumor-bearing nude mice, which was due to the strong binding of cRGD to the overexpressed integrin $\alpha_{v} \beta_{3}$ on the tumor cells. Moreover, the cRGDmodified DOX-loaded liposomes exhibited a significantly higher inhibitory effect on the growth of A549 tumor in nude mice, as compared with the linear RGD-modified liposome group or non-modified liposome group. Khabazian et al also investigated a cRGD-modified liposome. ${ }^{30}$ They found that the incorporation of cRGD peptide could increase the cytotoxicity of anti-STAT3 siRNA-loaded liposomes in B16F10 melanoma cells in vitro and enhance the accumulation of the liposomes in the B16F10 tumors in vivo. Ren et al reported that the incorporation of RGD could not only increase the liposome accumulation in the tumor but also enhance the endocytosis of liposomes by HRT-18 cells, which accounted for the increased antitumor effect of DOXloaded liposome in HRT-18 tumor-bearing mice. ${ }^{31}$ Based on these previous studies, we hypothesized that the incorporation of cRGD into the liposomal (-)-gossypol could significantly enhance the tumor-targeting effect and consequently improve the antitumor efficacy of this drug in vivo. In the present study, the physicochemical properties of (-)-gossypol-loaded cRGD-LP (Gos/cRGD-LP) were investigated in terms of particle size, zeta-potential, morphology, drug release profile, and so on. The inhibitory effect of Gos/cRGD-LP on the proliferation of tumor cells in vitro was evaluated using different cell lines. The biodistribution of the cyclic RGD-decorated liposomes in vivo was investigated via the near-infrared (NIR) fluorescence imaging technique. For the first time, our previously established quantitative analysis methods for NIR fluorescence images were adopted to evaluate the tumortargeting ability of the liposomes. Finally, the antitumor effect of Gos/cRGD-LP in vivo was evaluated in PC-3 tumor-bearing nude mice. For the above studies, a noncRGD-containing liposome (LP), as a passive targeting nanocarrier control for cRGD-LP, was also prepared and investigated for comparison.

\section{Materials and Methods Preparation of Gos/cRGD-LP}

Gos/cRGD-LP was prepared using combined methods of thin-film hydration and sonication. ${ }^{25}$ Briefly, (-)-gossypol $(9 \mathrm{mg})$ and the loading materials $(120 \mathrm{mg}$ in total) were added into $5 \mathrm{~mL}$ chloroform. The loading materials consisted of egg L- $\alpha$-phosphatidylcholine (EPC), cyclic RGDyk (Arg-Gly-Asp-d-Tyr-Lys) peptide-decorated $\mathrm{PEG}_{2000}$-DSPE (cRGD-PEG-DSPE, as shown in Figure 1B), and cholesterol at a molar ratio of 21:2:5. The solution was stirred for $30 \mathrm{~min}$ to dissolve all the solutes thoroughly and was preliminarily dried using a YRE-2000B rotary evaporator (Gongyi Yuhua Instrument, China) to form a thin film of (-)-gossypol /carrier mixture. The mixture film was then dried under nitrogen flow overnight to remove residual chloroform. Subsequently, the film was hydrated using $6 \mathrm{~mL}$ phosphate-buffered solution (PBS, $\mathrm{pH}$ 7.4) and was sonicated intermittently using a BIO-1800 probe sonicator (Shanghai Bonuo Biotechnology, China) for 6 min (net sonication time) at $160 \mathrm{~W}$ to produce a limpid suspension of Gos/ cRGD-LP (Figure 1C). Blank liposome (cRGD-LP) was prepared similarly without (-)-gossypol. The non-cRGDcontaining drug-loaded liposome (Gos/LP) was prepared similarly using PEGylated 1,2-distearoyl-sn-glycero -3-phosphoethanolamine (mPEG-DSPE, with the 
molecular weight of 2000 Da for mPEG) instead of cRGD-PEG-DSPE. The ${ }^{1} \mathrm{H}$ NMR spectra of cRGD, mPEG-DSPE, and cRGD-PEG-DSPE that confirmed their chemical structures are shown in Figure S1. DiR (1,1'-dioctadecyl-3,3,3',3'-tetramethylindotricarbocyanine iodide)-loaded liposomes (DiR/cRGD-LP and DiR/LP) for NIR fluorescence imaging studies were prepared similarly using $0.3 \mu \mathrm{mol}$ DiR instead of (-)-gossypol.

\section{Drug Concentration and Drug Loading Content (DLC)}

The drug concentrations of Gos/cRGD-LP samples were determined using a D-8PC UV-vis spectrophotometer (Nanjing Feile Instrument, China) at $385 \mathrm{~nm}$ wavelength. For stability studies, the drug concentrations of the samples stored for over 10 days at room temperatures (RT, 20 $\pm 2{ }^{\circ} \mathrm{C}$ ) were measured. The DLC of Gos/cRGD-LP was investigated via an ultrafiltration method. ${ }^{25}$ Gos/cRGDLP sample was transferred into an ultrafiltration centrifuge tube (MWCO $3000 \mathrm{Da}$ ), followed by centrifugation at $2800 \times g$ for $5 \mathrm{~min}$. The drug concentration in the filtrate, which indicated the concentration of free (-)gossypol in the Gos/cRGD-LP sample, was determined using the spectrophotometer. The DLC was then calculated using the formula: DLC $(\mathrm{wt} \%)=$ (weight of $(-)$ gossypol in the liposomes $\div$ total weight of drug-loaded liposomes $) \times 100 \%$. In addition, the drug loading efficiency (DLE) of Gos/cRGD-LP was calculated using the formula: DLE $(\mathrm{wt} \%)=$ (weight of (-)-gossypol in the liposomes $\div$ total weight of input (-)-gossypol) $\times 100 \%$. The above studies were similarly done on Gos/LP for comparison.

\section{Particle Size Distribution, Zeta-Potential, and Morphology}

The particle size distribution and zeta-potential of Gos/ cRGD-LP were investigated on a Nano ZS Zetasizer (Malvern Instruments, UK) using the matched sample cuvettes. $^{25}$ For morphology study, the suspension of Gos/ cRGD-LP was dropped on a carbon-coated copper grid for transmission electron microscopy (TEM) sample preparation. After negative-staining using uranyl acetate and natural drying, the sample was visualized on a Tecnai G2 transmission electron microscope (FEI Company, USA). The above studies were similarly done on Gos/LP for comparison.

\section{Release Profile of (-)-Gossypol from Gos/ CRGD-LP}

The release profile of (-)-gossypol from Gos/cRGD-LP in vitro was studied using a dynamic dialysis method under sink conditions. ${ }^{32}$ The suspension of Gos/cRGD-LP (6 mL) was transferred into a dialysis tube (MWCO $2000 \mathrm{Da}$ ) and was dialyzed against $400 \mathrm{~mL}$ PBS (pH 7.4) at $37^{\circ} \mathrm{C}$. During the dialysis, the dissolution medium was stirred at a speed of $80 \mathrm{rpm}$. At each predetermined time point, $1 \mathrm{~mL}$ of the dissolution medium was withdrawn; meanwhile, the same amount of fresh PBS was replenished. The released drug in the dissolution medium was extracted twice using chloroform. The total extraction recovery of (-)-gossypol from the dissolution medium was about $98.5 \%$. For each time, the dissolution medium was added with $1 \mathrm{~mL}$ chloroform. After vortexing for over $8 \mathrm{~min}$, the mixture was centrifuged at $6500 \times g$ for 10 min, and the clear chloroform layer (the extracting solution) was transferred into a clean test tube. After extracting twice, the extracting solutions were combined and mixed by vortexing. Then, $1.6 \mathrm{~mL}$ of the combined solution was withdrawn and was dried under nitrogen flow. The dry residue was dissolved using acetonitrile and filtered through a $0.22 \mu \mathrm{m}$ membrane, followed by the determination of the (-)-gossypol amount via high-performance liquid chromatography (HPLC). ${ }^{2}$ The HPLC analysis was conducted at $26{ }^{\circ} \mathrm{C}$ on a 1260 Infinity II HPLC system (Agilent Technologies, Inc., USA) which was equipped with a $\mathrm{C} 18$ reversed-phase column ( $5 \mu \mathrm{m}$ particle size, $4.6 \times 250 \mathrm{~mm}$ ) and a G7114A variable wavelength detector. Each sample $(20 \mu \mathrm{L})$ was separated using acetonitrile- $0.9 \%$ formic acid solution $(77: 23, \mathrm{v} / \mathrm{v})$ as the mobile phase $(0.8 \mathrm{~mL} / \mathrm{min})$, and was detected at $254 \mathrm{~nm}$ wavelength. The above studies were similarly done on Gos/ LP and free (-)-gossypol for comparison. To prepare a solution of free drug, $9 \mathrm{mg}(-)$-gossypol was dissolved in $6 \mathrm{~mL}$ PBS (pH 7.4) that contained $3 \%$ dimethyl sulfoxide (DMSO) as a solubilizer.

\section{In vitro Cytotoxicity}

The cytotoxicity of Gos/cRGD-LP in vitro was investigated in PC-3 (prostate cancer) and HCT-116 (colon cancer) cells. Both PC-3 and HCT-116 cell lines used in this study were purchased from Fuxiang Biotechnology Co., Ltd (Shanghai, China). The cells were incubated in 96well flat-bottomed plates $\left(1 \times 10^{4}\right.$ cells/well $)$ overnight at $37{ }^{\circ} \mathrm{C}$ before being treated with Gos/cRGD-LP of different (-)-gossypol concentrations. After incubation for $72 \mathrm{~h}$, Cell Counting Kit-8 (CCK-8) solution was added to each well 
according to the manual, and the cells were incubated for another 2 hours. Then, the cell viability was determined based on the absorbance which was measured at $450 \mathrm{~nm}$ wavelength using a Multiskan FC microplate reader (Thermo Fisher Scientific, USA). The above studies were similarly done on cRGD-LP, Gos/LP, and free drug for comparison.

\section{Animals}

BALB/c nude mice (male, $8-9$ weeks) and BALB/c mice (male, 8-9 weeks) were supplied by Charles River Laboratories (USA) and were housed under pathogenfree conditions with free access to food and water. Studies involving animal use were approved by the Ethics Committee of Southwest Medical University (approval number: 2020085) and complied with the NIH Guide for the Care and Use of Laboratory Animals.

\section{Maximum Tolerated Dose (MTD)}

The MTD of Gos/cRGD-LP or Gos/LP was studied via an observation method using BALB/c mice. ${ }^{25}$ The mice (22$26 \mathrm{~g})$ were randomly divided into eight groups $(\mathrm{n}=5)$ and were slowly injected ( $<1 \mathrm{~mL} / \mathrm{min}$, i.v.) with Gos/cRGD-LP or Gos/LP at doses of 34, 36, 38, and $40 \mathrm{mg}$ (-)-gossypol/kg. After the administrations, the survival and the changes in general appearance and body weight of each animal were observed for 10 days. MTD was determined as the highest dose not resulting in death, significant changes in the appearance, or $>15 \%$ body weight loss of the animals. ${ }^{25}$

\section{Animal Tumor Models}

The animal tumor models were established using the $\mathrm{BALB} / \mathrm{c}$ nude mice according to the reported methods. ${ }^{33}$ Briefly, the nude mice were s.q. injected with PC-3 cells suspended in Dulbecco's modified Eagle's medium (DMEM) via a sterile 23-gauge needle. For NIR fluorescence imaging studies, a $200 \mu \mathrm{L}$ suspension containing about $1 \times 10^{6}$ cells was injected on both sides (near the hind legs) of each mouse for tumor growth. For drug efficacy studies, a $200 \mu \mathrm{L}$ suspension containing $1 \times 10^{6}$ cells was injected on the back of each mouse. When the tumors in the mice reached predetermined volumes, the mice were used for in vivo experiments. The tumor volume was calculated using the formula: tumor volume $\left(\mathrm{mm}^{3}\right)=A \times B^{2} / 2$, in which "A" and "B" represented the longest and shortest diameters of the tumor, respectively.

\section{NIR Fluorescence Imaging}

The tumor-targeting effects of the liposomes in vivo were investigated via NIR fluorescence imaging. ${ }^{33}$ The PC-3 tumor-bearing mice were randomly divided into three groups $(\mathrm{n}=3)$. Each mouse was i.v. injected with $200 \mu \mathrm{L}$ suspension of DiR/cRGD-LP or DiR/LP that contained $10 \mathrm{nmol} \mathrm{DiR}$ or $200 \mu \mathrm{L}$ ethanol/PBS $(1: 4, \mathrm{v} / \mathrm{v})$ that contained $10 \mathrm{nmol}$ free $\mathrm{DiR}$ as a control. At each predetermined time point, the distribution of DiR/cRGD-LP, DiR/LP, or free DiR in the mouse was observed in an MS FX PRO imaging system (Carestream Health, USA), using the excitation wavelength of $750 \mathrm{~nm}$ and emission wavelength of $830 \mathrm{~nm}$. For each imaging, the f-stop was 2.8 , and the exposure time was $60 \mathrm{~s}$. The imaging settings were fixed for the entire imaging experiment. At $72 \mathrm{~h}$ post-injection, the mice were euthanized, and their tumors and organs/tissues were used for ex vivo imaging, using the same settings as for in vivo imaging.

\section{Quantitative Analysis of NIR}

\section{Fluorescence Imaging}

To better evaluate the tumor-targeting effects, previously reported quantitative evaluation methods for NIR fluorescence imaging were adopted. ${ }^{33}$ The fluorescence intensity of the whole body and the tumor regions in the photos from in vivo imaging and the tumors and organs in the photos from ex vivo imaging were quantified using the "Manual Regions of Interest" function of the matched Carestream imaging software (Carestream Health, USA). Then, the tumortargeting index (TTI) of each sample at each time point during in vivo imaging was calculated using the formula: TTI $(\%)=$ (fluorescence intensity of tumor region $\div$ fluorescence intensity of whole body) $\times 100 \%{ }^{33}$ Furthermore, the area under the TTI-time curve (AUTC) was calculated based on the trapezoidal rule according to the previous studies. ${ }^{33,34}$ For ex vivo imaging, the fluorescence intensity ratios of tumor to organs (ie liver, spleen, or lung) were calculated using the formula: Intensity ratio $=$ fluorescence intensity of tumor $\div$ fluorescence intensity of organ.

\section{In vivo Antitumor Effects}

When the tumor volume reached $50 \mathrm{~mm}^{3}$ on average, the tumor-bearing nude mice were randomly divided into five groups $(\mathrm{n}=8)$. The mice in different groups were treated as follows: (1) PBS (i.v., blank control), (2) cRGD-LP (i.v., vehicle control), (3) free (-)-gossypol (suspension in PBS, p.o., $15 \mathrm{mg} / \mathrm{kg}$ ), (4) Gos/LP (i.v., $15 \mathrm{mg}$ (-)-gossypol/kg), and (5) Gos/cRGD-LP (i.v., $15 \mathrm{mg}$ (-)-gossypol/kg). The 
treatments were done once every three days, for 6 times in total. The tumor volumes and the body weights of the mice were measured once every three days, until the end of observation. The tumor growth inhibition rate (IR) of each group as compared with the PBS group was calculated using the formula: IR $(\%)=(1-T / C) \times 100 \%$, in which " $\mathrm{T}$ " is the average tumor volume of a test group, and " $\mathrm{C}$ " is the average tumor volume of the PBS group measured on the same day. ${ }^{33}$ For the terminal deoxynucleotidyl transferase-mediated dUTP-biotin nick-end labeling (TUNEL) analysis, the excised tumors from different groups were fixed in $4 \%$ paraformaldehyde solution and were then embedded with paraffin and sectioned. TUNEL staining was performed on each tumor section using TUNEL apoptosis assay kits (Beyotime Biotechnology, Shanghai, China), followed by observation of the stained sections on an Eclipse Ti-S microscope (Nikon, Japan).

\section{Changes in Plasma Potassium $\left(\mathrm{K}^{+}\right)$Level}

To study the potential effects of (-)-gossypol on the plasma $\mathrm{K}^{+}$level, BALB/c nude mice were randomly divided into four groups $(n=8)$. One of the groups was not given any treatment (the control). The mice in the other three groups were treated according to the above study of antitumor effects, ie: (1) free (-)gossypol (suspension in PBS, p.o., $15 \mathrm{mg} / \mathrm{kg}$ ), (2) Gos/ LP (i.v., $15 \mathrm{mg}$ (-)-gossypol/kg), and (3) Gos/cRGD-LP (i.v., $15 \mathrm{mg}(-)$-gossypol/kg). The treatments were done once every three days, for 6 times in total. One day after the last treatment, a blood sample $(0.8-1.0 \mathrm{~mL})$ was collected from the heart of each mouse and was centrifuged $(2800 \times g$ for $10 \mathrm{~min})$ to obtain the plasma. The plasma $\mathrm{K}^{+}$concentration was determined on an AU5800 biochemistry analyzer (Beckman Coulter, USA), using the matched reagent and calibration solution for the equipment. 2,35

\section{Statistical Analysis}

The data were analyzed and exhibited using Prism 5 software (GraphPad Software, USA). ${ }^{34,36}$ The comparison of data was done using Student's $t$-test (between two groups) and One-way ANOVA (among multiple groups). The $P$ value $<0.05$ was considered statistically significant. The $\mathrm{IC}_{50}$ values for in vitro cytotoxicity were calculated based on the cell viability using the "nonlinear regression" function of the GraphPad Prism 5 software.

\section{Results and Discussion Design and Preparation of Gos/cRGD-LP} For nanocarriers designed based on the tumor cell targeting strategy, the need for extravasation of the nanocarriers from tumor vasculature into tumor tissue is one of the major factors reducing the targeting effect. ${ }^{27}$ Moreover, specific cell-surface receptors vary among different tumor types, which can limit the application of tumor celltargeted nanocarriers. By contrast, nanocarriers targeting tumor vasculature can bind to the receptors on vascular endothelial cells directly after i.v. injection, and most of these receptors, including vascular endothelial growth factor receptors (VEGFRs), $\alpha_{\mathrm{v}} \beta_{3}$ integrin, and vascular cell adhesion molecule-1 (VCAM-1), etc., are over-expressed in various tumor types. ${ }^{27}$ Integrins are transmembrane heterodimers working as cell adhesion and signaling receptors on the cells that mediate important physiological processes (eg embryogenesis and angiogenesis) and pathophysiological processes (eg vascularization, cell growth, and metastasis of tumors) through their interaction with the extracellular matrix (ECM). ${ }^{37,38}$ The tripeptide ArgGly-Asp (RGD) is the highly conserved recognition motif in the ECM proteins toward some of the integrins (ie the RGD-binding integrins as one subfamily of the integrins).$^{37}$ Most of the RGD-binding integrins are expressed at increased levels in various cancer types and play essential pathophysiological roles in cancer progression. The integrin $\alpha_{v} \beta_{3}$ is one of the RGD-binding integrins. During tumor angiogenesis, integrin $\alpha_{v} \beta_{3}$ is highly upregulated on angiogenic endothelial cells and mediates the interaction of activated endothelial cells with the ECM. On tumor cells of many cancer types such as prostate cancer, gastric cancer, breast cancer, pancreatic cancer, and glioma, integrin $\alpha_{v} \beta_{3}$ is overexpressed during tumor progression. The interaction of integrin $\alpha_{\mathrm{v}} \beta_{3}$ with the ECM through RGD binding is involved in the regulation of cell mechanical outcomes that have crucial effects on the adhesion, migration, and proliferation of cancer cells. ${ }^{38}$ Since integrin $\alpha_{\mathrm{v}} \beta_{3}$ is overexpressed on both tumor vasculature and tumor cells, it has great potential for targeted cancer therapy. ${ }^{39}$

In the present study, cRGDyk with high affinity to $\alpha_{\mathrm{v}} \beta_{3}$ integrin was introduced to a mixed liposome (cRGD-LP) for enhanced targeting effect that may improve the antitumor effect of (-)-gossypol in vivo (Figure 1C). In our pilot study, the input molar ratio of the loading materials (ie EPC, cRGD-PEG-DSPE, and cholesterol at 21:2:5) 
was roughly optimized to achieve a relatively smaller particle size, higher DLC, and moderate drug release rate. EPC is one of the most used lipids for liposome preparation and was the main component for cRGD-LP of this study. ${ }^{40} \mathrm{EPC}$ is biocompatible and can form liposomes with an approximately neutral surface charge that is favorable for the evasion of renal elimination. ${ }^{27}$ Cholesterol is a small lipid-type molecule that plays essential physiological roles in the body. ${ }^{41}$ It has been reported to be able to stabilize liposomal formulations by avoiding the phase transition of the phospholipids. ${ }^{42}$ Moreover, due to its significant hydrophobicity, incorporation of cholesterol into liposome may result in better hydrophobic interactions between the loading materials and (-)-gossypol, which is a hydrophobic drug, thus improving the drug loading efficiency and achieving higher drug concentrations for injection. However, stronger hydrophobic interactions may also retard the release of (-)-gossypol from the liposomes. Therefore, the input amount of cholesterol should be optimized to achieve acceptable drug loading efficiency and a proper drug release rate.

The tumor-targeting ligand cRGD was incorporated into the liposome by using cRGD-PEG-DSPE. It is noteworthy that the input molar ratio of these loading materials can be further modulated according to the tumor-targeting effect of the liposome. Moreover, the comparative experiments for cRGD-LP were carefully designed. Using mPEG-DSPE to replace cRGD-PEG-DSPE, a non-cRGDcontaining liposome (LP) as a passive targeting nanocarrier control was prepared. Except for the cRGD portion, cRGD-PEG-DSPE and mPEG-DSPE had very similar chemical structures (Figure 1B), which made LP a specialized control for cRGD-LP.

\section{Characteristics of Gos/cRGD-LP}

The DLC and DLE were about $6.46 \%$ and $92.10 \%$, respectively, for Gos/cRGD-LP and were about $6.39 \%$ and $91.08 \%$, respectively, for Gos/LP (Table 1). The results between Gos/cRGD-LP and Gos/LP were not significantly different $(p>0.05)$. The drug concentrations of both the formulations were about $1.5 \mathrm{mg}(-)$-gossypol $/ \mathrm{mL}$. The particle size of Gos/cRGD-LP was $61.86 \pm 1.73 \mathrm{~nm}$, which was either not significantly different $(p>0.05)$ from that (ie $59.06 \pm 1.81 \mathrm{~nm}$ ) of Gos/LP (Table 1 and Figure 2A). The particle sizes within the range of 10-100 $\mathrm{nm}$ were usually ideal for the passive targeting effect of nanocarriers via the enhanced permeability and retention (EPR) effect. ${ }^{27}$ Moreover, the polydispersity index (PDI)
Table I Summary of the Characteristics of Gos/LP and Gos/ cRGD-LP. Data are Shown as Mean \pm SD $(n=3)$

\begin{tabular}{|l|l|l|}
\hline Formulation & Gos/LP & Gos/cRGD-LP \\
\hline DLC (\%) & $6.46 \pm 0.09$ & $6.39 \pm 0.04$ \\
DLE (\%) & $92.10 \pm 1.34$ & $91.08 \pm 0.60$ \\
Drug concentration $(\mathrm{mg} / \mathrm{mL})$ & $1.501 \pm 0.028$ & $1.498 \pm 0.020$ \\
Particle size $(\mathrm{nm})$ & $59.06 \pm 1.8 \mathrm{I}$ & $61.86 \pm 1.73$ \\
PDI & $0.126 \pm 0.012$ & $0.118 \pm 0.009$ \\
Zeta-potential $(\mathrm{mV})$ & $0.90 \pm 0.89$ & $-3.53 \pm 1.78$ \\
\hline
\end{tabular}

of $<0.2$ for both the formulations indicated a homogenous population of liposomes. ${ }^{43}$ The zeta-potential was around $0 \mathrm{mV}$ for Gos/LP but was slightly negative for Gos/cRGDLP (Table 1 and Figure 2B). The negative surface charge of Gos/cRGD-LP was probably caused by the ionization of amino acids in cRGD under weakly alkaline conditions (pH 7.4). The TEM photos of Gos/LP and Gos/cRGD-LP are shown in Figure 2C. For both the formulations, small liposomes showed similar spherical shapes with no obvious difference between the two samples. Compared with the smaller liposomes, the larger liposomes were generally less spherical, and some of them even had invagination. The invagination of liposomes can occur during the sample preparation for TEM, because the water in the liposomes may be driven out after the drying process. ${ }^{44}$ For stability studies, the drug concentration, particle size, and zeta-potential of Gos/cRGD-LP and Gos/LP stored for over 10 days at RT $\left(20 \pm 2{ }^{\circ} \mathrm{C}\right)$ were measured. As a result, the drug concentrations of both the formulations were still about $1.5 \mathrm{mg}$ (-)-gossypol/mL (1.491 $\pm 0.017 \mathrm{mg}(-)$ gossypol/mL for Gos/cRGD-LP, and $1.489 \pm 0.021 \mathrm{mg}(-)-$ gossypol/mL for Gos/LP), with no drug precipitation observed. After the storage, the particle size was $64.31 \pm$ $2.33 \mathrm{~nm}(\mathrm{PDI}=0.188 \pm 0.009)$ for Gos/cRGD-LP and was $61.01 \pm 2.43 \mathrm{~nm}(\mathrm{PDI}=0.191 \pm 0.014)$ for Gos/LP. The zeta-potential was still around $0 \mathrm{mV}$ for Gos/LP and was slightly negative for Gos/cRGD-LP. These results indicated the good stability of Gos/cRGD-LP and Gos/LP. It is noteworthy that, for nanocarrier that is produced based on a thin-film hydration method, the thin film of loading materials/drug mixture before hydration can be considered for long-term storage (ie for over 6 months). Because as a solid intermediate product of nanomedicine, the film of loading materials/drug mixture can maintain stability for a much longer time under suitable conditions (eg in a nitrogen atmosphere), as compared with the final nanomedicine suspended in water. $^{32}$ In such a strategy, the 
A

Gos/LP

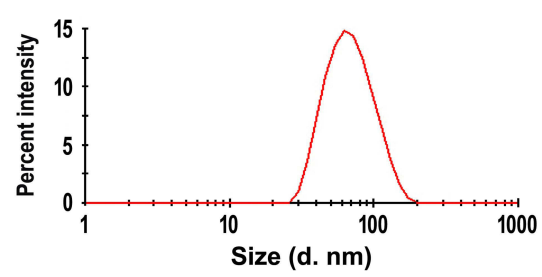

B

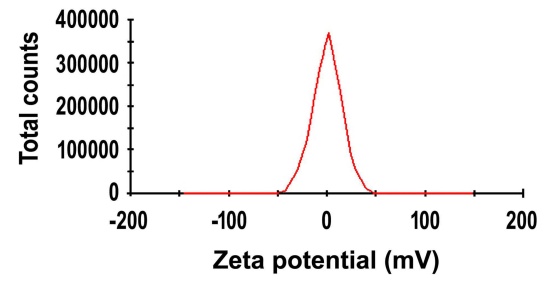

C

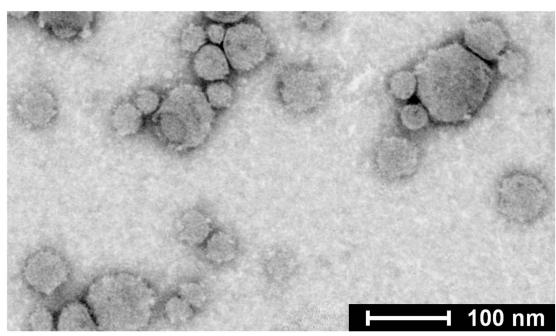

Gos/cRGD-LP
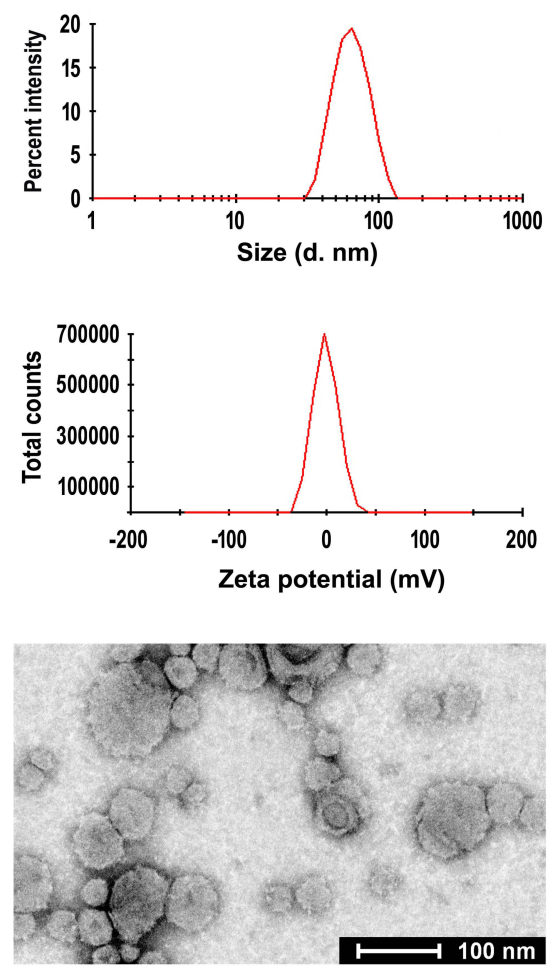

Figure 2 (A) Particle size distribution, (B) zeta potential, and (C) TEM images of DiR/LP and DiR/cRGD-LP.

stability of Gos/cRGD-LP and Gos/LP may be hopefully maintained for a long time.

\section{Drug Release of Gos/cRGD-LP}

Figure 3 exhibits the release profiles of (-)-gossypol from Gos/cRGD-LP or Gos/LP in vitro as studied using a dialysis method. The diffusion of the free drug from the dialysis tube was fast, with over $83 \%$ of the drug diffused into the dissolution medium within the first $12 \mathrm{~h}$ (the free (-)gossypol group in Figure 3). By contrast, Gos/cRGD-LP and Gos/LP showed much slower release, with no burst release of (-)-gossypol. The drug release profiles for the two formulations were similar, probably because the main building blocks (ie EPC and cholesterol) were the same for cRGD-LP and LP. Under the experimental conditions of this study, Gos/cRGD-LP and Gos/LP showed sustained drug release for over $96 \mathrm{~h}$ in vitro (Figure 3).

\section{In vitro Cytotoxicity}

The cytotoxicity of free (-)-gossypol, Gos/LP, Gos/cRGD-LP, and blank cRGD-LP against PC-3 (prostate cancer) and HCT116 (colon cancer) cells was investigated. For PC-3 cells, the $\mathrm{IC}_{50}$ of free (-)-gossypol was $8.72 \pm 0.30 \mu \mathrm{M}$ (Figure 4A).
Compared with the free drug, Gos/LP showed a significantly higher $\mathrm{IC}_{50}$ of $10.68 \pm 0.53 \mu \mathrm{M}(\mathrm{p}<0.01)$, which may result from the sustained drug release of the liposomes. $\mathrm{The}^{\mathrm{IC}} \mathrm{C}_{50}$ of

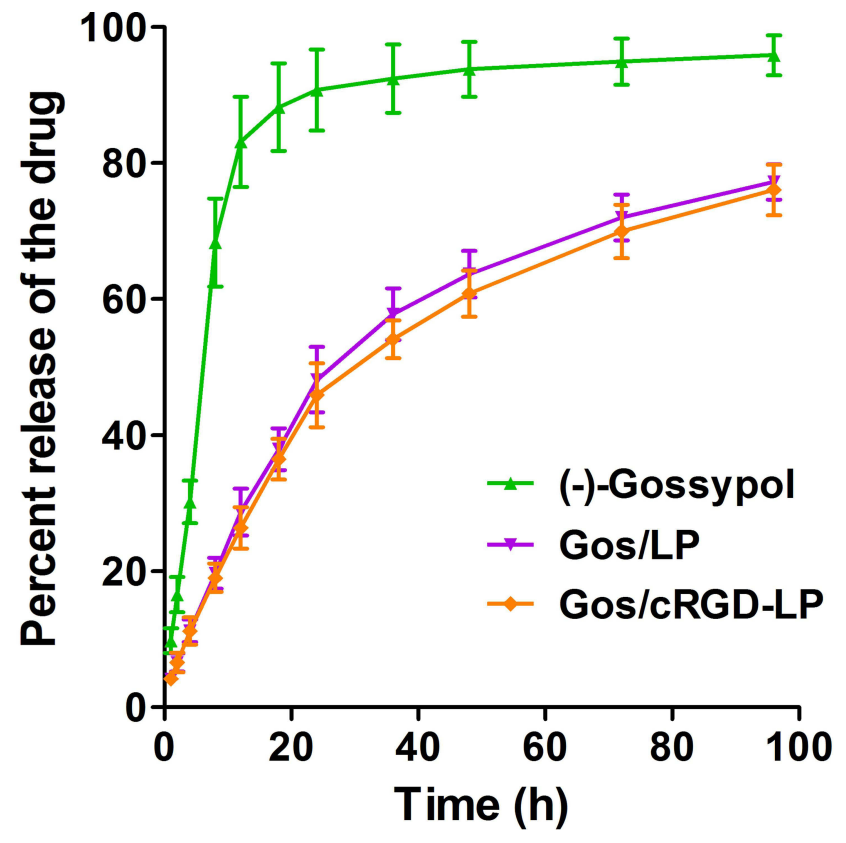

Figure 3 In vitro release profiles of (-)-gossypol from DiR/LP and DiR/cRGD-LP in PBS at $37^{\circ} \mathrm{C}$. Data are shown as mean \pm SD $(n=3)$. The two formulations showed similar drug release profiles. 
Gos/cRGD-LP was $9.53 \pm 0.26 \mu \mathrm{M}$, also higher than that of free $(-)$-gossypol $(p<0.05)$. However, it is interesting to find that the $\mathrm{IC}_{50}$ of Gos/cRGD-LP was significantly lower than that of Gos/LP $(p<0.05)$. The increased cytotoxicity of Gos/ cRGD-LP as compared with Gos/LP was probably due to the enhanced endocytosis of Gos/cRGD-LP induced by the binding of cRGD with $\alpha \mathrm{v} \beta 3$ integrin on PC-3 cells.

For HCT-116 cells, the $\mathrm{IC}_{50}$ of free (-)-gossypol was 6.32 $\pm 0.40 \mu \mathrm{M}$ (Figure 4B). By contrast, the $\mathrm{IC}_{50}$ values for Gos/ LP $(9.03 \pm 0.45 \mu \mathrm{M})$ and Gos/cRGD-LP $(8.93 \pm 0.41 \mu \mathrm{M})$ were both significantly increased $(\mathrm{p}<0.01)$, which was in accordance with the results of PC-3 cells. Nevertheless, for HCT-116 cells, although Gos/cRGD-LP showed higher cytotoxicity than Gos/LP, the difference between the two formulations was not statistically significant $(p>0.05)$. It should be noted that HCT-116 cells overexpress $\alpha_{v} \beta_{5}$ integrin rather than $\alpha_{v} \beta_{3}$ integrin. ${ }^{45}$ It has been reported that the affinity of cRGDyk to $\alpha_{v} \beta_{5}$ integrin was much weaker than to $\alpha_{v} \beta_{3}$ integrin. ${ }^{46}$ Therefore, in some previous studies on nanocarriers targeting HCT-116 cells, cRGD proteins (eg cRGDfC) with high affinity to $\alpha_{v} \beta_{5}$ integrin were used rather than cRGDyk. ${ }^{47-49}$ Based on the possible reasons above, it is not strange to observe similar cytotoxicity for Gos/LP and Gos/cRGD-LP against HCT-116 cells. However, more evidence is needed to confirm such a conjecture. The cytotoxicity of the blank cRGD-LP at different concentrations was
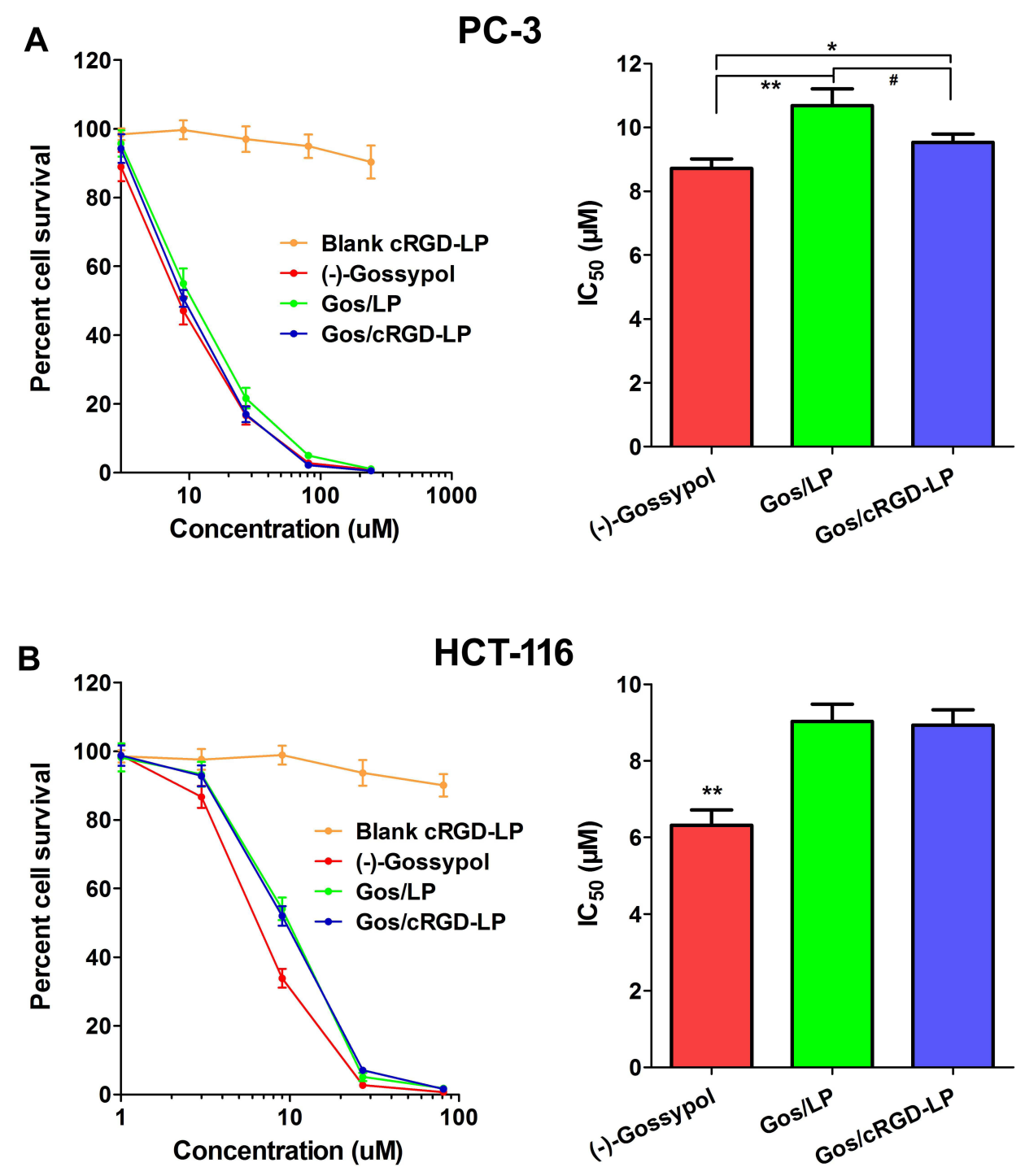

Figure 4 The viability of (A) PC-3 (prostate cancer) and (B) HCT-II6 (colon cancer) cells treated with free (-)-gossypol, Gos/LP, Gos/cRGD-LP, and blank cRGD-LP. Data are shown as mean \pm SD ( $n=3)$. For PC-3 cells, free (-)-gossypol showed lower IC50 as compared with Gos/LP (**p <0.0I) and Gos/cRGD-LP (*P <0.05); Gos/cRGD-LP showed lower IC50 as compared with Gos/LP (\#p < 0.05). For HCT-II 6 cells, free (-)-gossypol showed lower IC50 as compared with Gos/LP and Gos/cRGD-LP (**P < 0.0I); the difference between Gos/LP and Gos/cRGD-LP was not statistically significant $(p>0.05)$. 
also evaluated (Figure 4). For both PC-3 and HCT-116 cells, cRGD-LP showed very limited cytotoxicity even at its highest concentration (equal to the concentration of the loading materials in Gos/cRGD-LP), which indicated good biocompatibility of the nanocarrier.

\section{MTD of Gos/cRGD-LP}

The MTD of Gos/cRGD-LP in mice was studied and compared with Gos/LP. Four dose levels (ie 34, 36, 38, and $40 \mathrm{mg}$ (-)-gossypol/kg) were tested for single i.v. injection. The survival and the changes in general appearance and body weight of each animal were observed for 10 days. As a result, for both the formulations at all tested dose levels, neither death nor significant changes in the general appearance of the animals has been observed (Table 2). However, the body weight loss generally became greater with increasing the dose level. For both the formulations, 2 of 5 mice have shown body weight losses of $>15 \%$ only at $40 \mathrm{mg}$ (-)-gossypol $/ \mathrm{kg}$. Therefore, according to the criteria for determining MTD, both Gos/ cRGD-LP and Gos/LP could be tolerated at up to $38 \mathrm{mg}(-)-$ gossypol/kg in mice. Nevertheless, the body weight loss at some lower dose levels should not be neglected (Table 2). For both the formulations, 3 of 5 mice had body weight losses of $>10 \%$ at $38 \mathrm{mg}(-)$-gossypol/kg. Moreover, for Gos/LP, 1 of 5 mice had a body weight loss of $>10 \%$ at $36 \mathrm{mg}$ (-)-gossypol $/ \mathrm{kg}$. The above results indicated that notable systemic toxicity may occur near the MTD. Although for single injection the body weights of the animals could recover later, for designing multiple-dose regimens the cumulative toxicity must be considered, and a dose level that is far below the MTD may be more suitable

\section{NIR Fluorescence Imaging}

The tumor-targeting effects of cRGD-LP and LP in vivo were investigated via NIR fluorescence imaging. PC-3 tumor-bearing nude mice were injected with DiR-loaded liposomes (ie DiR/cRGD-LP or DiR/LP) or free DiR dissolved in ethanol/PBS $(1: 4, \mathrm{v} / \mathrm{v})$ as the control. Each mouse bearing tumors on both sides (near the hind legs) was given the same amount (ie $10 \mathrm{nmol}$ ) of DiR whatever the formulation. Figure 5 shows the representative fluorescence images of mice at different time points after the injection. All the images were obtained under the same imaging settings for better comparison. At $6 \mathrm{~h}$ after the injection, a considerable fluorescence signal could be observed in the tumor regions of the mice receiving DiR/cRGD-LP and DiR/LP (Figure 5). After that, the fluorescence intensity in these tumors increased with time, which indicated the accumulation of the DiR-loaded liposomes in the tumors. Strong fluorescence intensity could be detected in the tumors for over two days. By contrast, the mice receiving free DiR showed very weak fluorescence signals in the body, which was probably caused by two reasons. Firstly, free DiR molecules in the blood can be rapidly cleared from the body, which has been observed in many other studies. ${ }^{24,26,50,51}$ The DiR molecules that have entered some tissues or organs such as the liver and spleen may remain in the body longer. Secondly, the fluorescence imaging was planar, the fluorescence signal from subcutaneous tumors and shallow organs/tissues that are near the camera could be well captured by the equipment, while that from deep organs/tissues in the body attenuated a lot when it reaches the camera. ${ }^{33}$ For this reason, ex vivo imaging of some deep organs/tissues is needed for a more comprehensive investigation of the fluorescence distribution. The fluorescence intensity kept weak in the tumor regions of the mice receiving free DiR throughout the in vivo imaging (Figure 5), indicating that the free DiR was not able to accumulate in the tumors on its own. It is noteworthy that for some of the photos the limbs of the mouse were not stretched enough and were under the body of the mouse during the imaging

Table 2 Maximum Tolerated Dose (MTD) of Gos/LP and Gos/cRGD-LP. Data Were Obtained from the Observation for 10 Days After the Injection

\begin{tabular}{|l|l|l|l|l|}
\hline Formulation & Dose (mg (-)-Gossypol/kg) & Mouse Death & Body Weight Loss > I0\% & Body Weight Loss > I5\% \\
\hline Gos/LP & 34 & $0 / 5$ & $0 / 5$ & $0 / 5$ \\
& 36 & $0 / 5$ & $1 / 5$ & $0 / 5$ \\
& 38 & $0 / 5$ & $3 / 5$ & $0 / 5$ \\
& 40 & $0 / 5$ & $4 / 5$ & $2 / 5$ \\
\hline Gos/CRGD-LP & 34 & $0 / 5$ & $0 / 5$ & $0 / 5$ \\
& 36 & $0 / 5$ & $0 / 5$ & $0 / 5$ \\
& 38 & $0 / 5$ & $3 / 5$ & $0 / 5$ \\
& 40 & $0 / 5$ & $5 / 5$ & $2 / 5$ \\
\hline
\end{tabular}



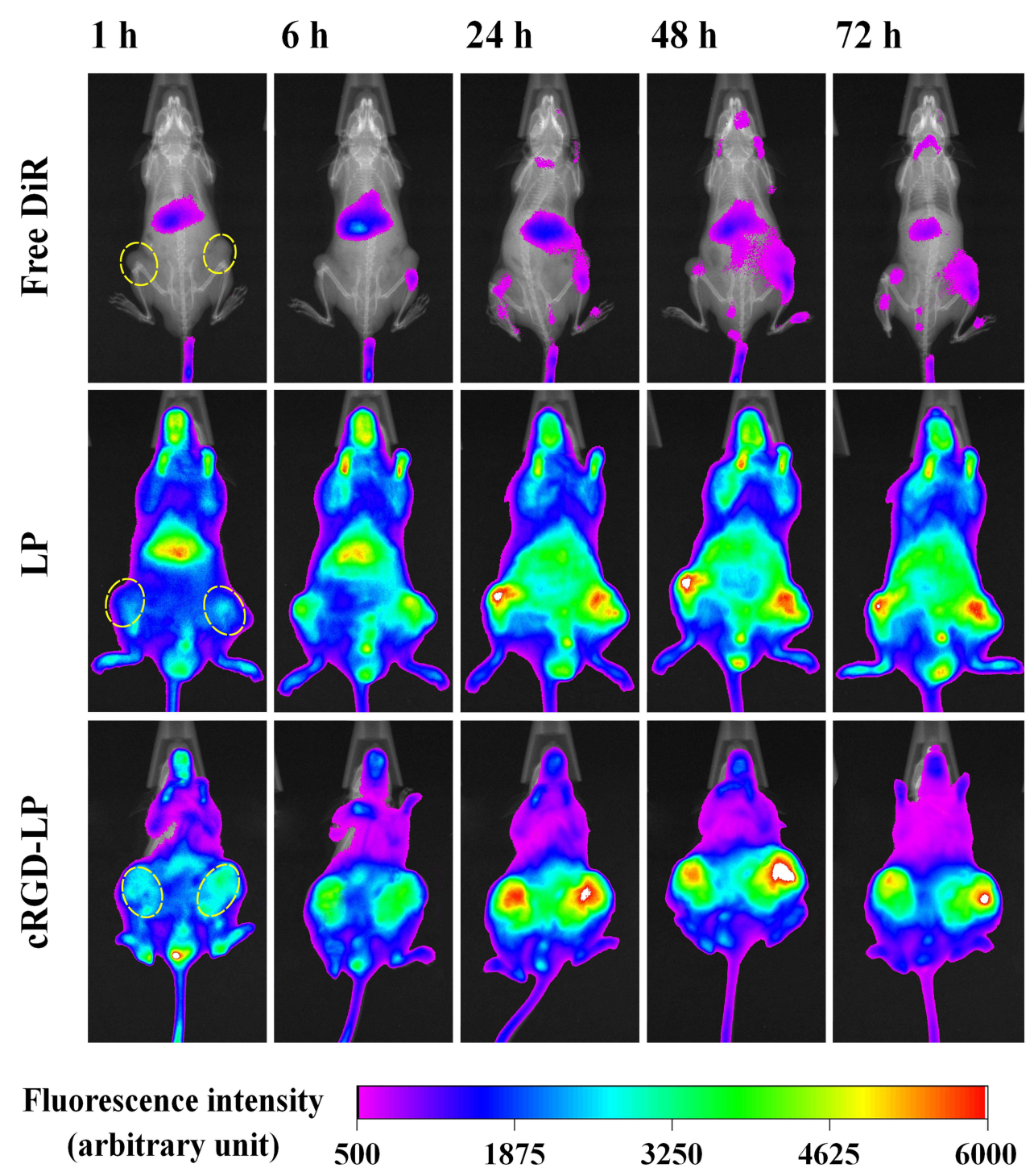

Figure 5 In vivo NIR fluorescence imaging of PC-3 tumor-bearing nude mice injected with DiR-loaded liposomes (cRGD-LP or LP) or free DiR dissolved in ethanol/PBS (I:4, v/v). Each mouse was given the same amount of DiR whatever the formulation. The fluorescence images were obtained at different time points after the injection under the same imaging settings. The tumor regions of each mouse at I $\mathrm{h}$ are indicated by yellow dash circles.

process (eg the photo for the cRGD-LP group at $48 \mathrm{~h}$ in Figure 5). Since the limbs did not shelter the tumor sites, the fluorescence from the tumor regions could be well observed and the analysis of the tumor fluorescence intensity was not affected in this study. Nevertheless, for future imaging studies, more attention should be paid to ensure that the limbs of the animals can be stretched enough for better observation and to avoid potential mistakes in analysis. Despite that considerable fluorescence intensity in the tumor regions was detected for cRGD-LP and LP, it was hard to determine which formulation had a better tumor-targeting effect according to the images because their results were generally similar. It is worth mentioning that, although in vivo NIR fluorescence imaging has been widely used to study the biodistribution of tumor-targeting nanocarriers, the evaluation of the targeting ability was mainly done intuitively based on images rather than objectively based on quantitative calculations. ${ }^{24,26,50-53}$ There has long been a lack of attention to developing quantitative methods for the evaluation of in vivo tumor-targeting effects. In this study, the tumortargeting effect of cRGD-LP and LP were quantitatively evaluated using our previously reported methods. ${ }^{33}$ Figure 6A exhibits the fluorescence intensity curves of the tumor regions of different groups. For all imaging time points, the tumor fluorescence of the LP group was significantly stronger than that of the free DiR group $(p<0.001$ at 1 , 6, 24, and $48 \mathrm{~h}$; and $\mathrm{p}<0.01$ at $72 \mathrm{~h}$ ), which was in accordance with the results of in vivo imaging. Compared 

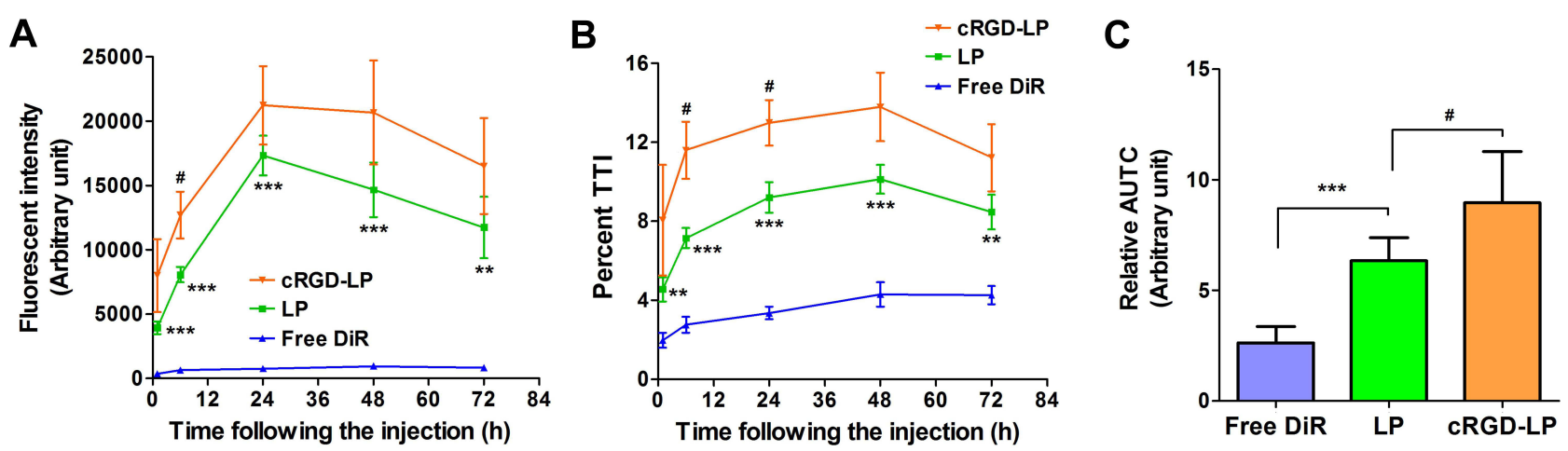

Figure 6 Quantitative analysis of in vivo NIR fluorescence imaging. (A) The DiR fluorescence intensity curves of the tumor regions in the mice receiving DiR-loaded liposomes (cRGD-LP or LP) or free DiR. (B) The tumor-targeting index (TTI) curves of different groups. (C) The relative area under the TTI-time curve (AUTC) of each group. Data are shown as mean \pm SD $(n=6)$. In figures $(\mathbf{A})$ and $(\mathbf{B})$, ${ }^{* *} p<0.0 \mathrm{I}$ and ${ }^{* * *} \mathrm{p}<0.00 \mathrm{I}$ for $L P$ as compared with free $\mathrm{DiR}$ at the same time points; \#p $<0.05$ for cRGD-LP as compared with LP at the same time points. In figure $(\mathbf{C})$, ${ }^{* * *} \mathrm{P}<0.00 \mathrm{I}$ for LP as compared with free DiR; \#p < 0.05 for cRGD-LP as compared with LP.

with LP, cRGD-LP showed even stronger tumor fluorescence for all time points, although only at $6 \mathrm{~h}$ the difference between the two groups was statistically significant $(p<$ 0.05 ). It should be noted that the tumor fluorescence intensity alone cannot indicate the specificity of the nanocarriers for tumors. Sometimes, strong tumor fluorescence was not due to the good tumor-targeting effect of the nanocarriers, but due to strong fluorescence intensity in the whole body. ${ }^{33}$ Therefore, the calculation of TTI values is a need to avoid misjudgment. Since the fluorescence intensity of the tumor region reflects the extent of drug accumulation in the tumors, a higher percent TTI indicates a better tumor-targeting ability of the nanocarriers. Nevertheless, each TTI value can only reflect the tumor-targeting ability at a single time point; thereby the calculation of AUTC was needed to indicate the overall tumor-targeting effect of the nanocarriers.

As shown in Figure 6B, the average TTI of cRGD-LP was higher than that of LP for all time points, although only at 6 and $24 \mathrm{~h}$ the difference between the two groups was statistically significant $(\mathrm{p}<0.05)$. These results are in accordance with Figure 6A. However, it is interesting to find that the maximal tumor fluorescence intensity appeared at $24 \mathrm{~h}$ for LP and cRGD-LP, while their TTI reached a climax at $48 \mathrm{~h}$. This result indicated that the liposomes may have the best tumor-targeting effects two days (rather than one day) after the injection. Moreover, despite that the tumor fluorescence intensity of the free DiR group was far weaker as compared with the LP group (Figure 6A), the average TTI of free DiR was over $4.26 \%$ at 48 and $72 \mathrm{~h}$, which was very near that of the LP group $(4.56 \%)$ at $1 \mathrm{~h}$. This result could hardly be discovered from the in vivo images (Figure 5). For better comparison, the area under the TTI-time curve (AUTC) was also calculated. It turned out that the AUTC of cRGD-LP was significantly larger than that of LP, suggesting a better overall tumor-targeting effect of cRGD-LP for the entire in vivo imaging process. ${ }^{33}$ Figure 7 shows the representative fluorescence images of tumors and organs/tissues from different groups. The cRGD-LP and LP groups showed comparable tumor fluorescence intensity notably stronger than that of the free DiR group, which was in accordance with the in vivo imaging results. Considerable fluorescence intensity could be observed in the liver, spleen, and lung of the free DiR group, which could hardly be discovered from the in vivo images (Figure 5). This result suggested that the free dye molecules tend to accumulate in the normal organs rather than in the tumors. The LP group also showed strong fluorescence in the liver. By
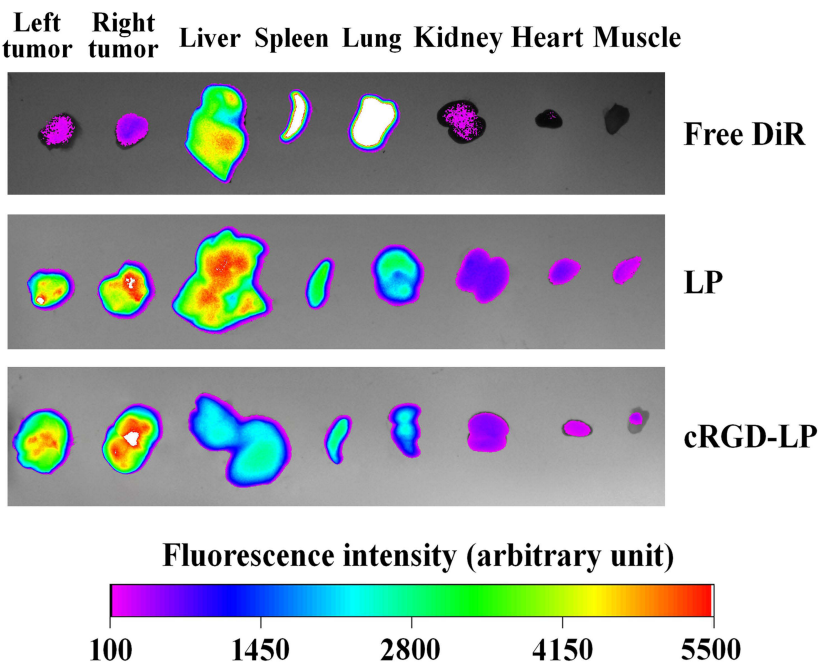

Figure 7 Ex vivo NIR fluorescence imaging of tumors and organs/tissues from the mice injected with DiR-loaded liposomes (cRGD-LP or LP) or free DiR. The tumors and organs were harvested at $72 \mathrm{~h}$ after the injection. The fluorescence images were obtained under the same imaging settings. 
contrast, cRGD-LP resulted in lower fluorescence intensity in the liver, spleen, and lung. The above results indicated that cRGD-LP may have more accumulation in the tumors and less accumulation in the normal organs/tissues as compared with free DiR and LP. For all groups, the fluorescence intensity of the kidney, heart, and muscle was lower as compared with that in the liver, spleen, and lung. For further comparison between cRGD-LP and LP, the fluorescence intensity of each tumor and organ/tissue was quantified. Among the three groups, cRGD-LP had the highest fluorescence intensity in the tumor and the lowest in the liver and lung; LP had the highest fluorescence intensity in the liver and kidney and the lowest in the spleen (Figure 8A). It turned out that cRGD-LP showed the highest fluorescence intensity ratios of tumor to liver, spleen, and lung, respectively, although the results of both cRGD-LP and LP groups were significantly better than those of the free DiR group (Figure $8 \mathrm{~B}-\mathrm{D}$ ). According to the above results of fluorescence imaging, cRGD-LP had a better tumor-targeting effect in vivo, which was probably due to the binding of cRGD with $\alpha_{\mathrm{v}}$ $\beta_{3}$ integrin on the tumor vascular endothelium and the tumor cells. ${ }^{27,39}$

\section{In vivo Antitumor Effects}

The antitumor effects of Gos/cRGD-LP and Gos/LP in vivo were investigated in PC-3 tumor-bearing mice. The mice were given PBS (i.v., blank control), blank cRGD-LP (i.v., vehicle control), free (-)-gossypol (p.o., $15 \mathrm{mg} / \mathrm{kg}$ ), Gos/LP (i.v., $15 \mathrm{mg}$ (-)-gossypol/kg), or Gos/cRGD-LP (i.v., $15 \mathrm{mg}(-)$ gossypol $/ \mathrm{kg}$ ). All the animals kept alive throughout the experiment. The tumor growth curves and the tumor inhibition rates of different groups were exhibited in Figure 9A and B, respectively. Under the experimental conditions of this study, the tumor volumes of the mice receiving PBS and blank cRGDLP increased rapidly, with no significant difference between the two control groups ( $\mathrm{p}<0.05$ for all time points). As a result, blank cRGD-LP showed negligible tumor inhibition rates of $<10 \%$ (Figure 9B). By contrast, the mice receiving the free drug or the drug-loaded liposomes (Gos/LP or Gos/ cRGD-LP) showed retarded tumor growth (Figure 9A). Nevertheless, compared with free (-)-gossypol, Gos/LP and Gos/cRGD-LP showed significantly improved inhibitory effects of tumor growth ( $\mathrm{P}<0.001$ on day 25), with the tumor inhibition rates of $>50 \%$ at the end of the experiment (from day 16 for Gos/cRGD-LP, and from day 19 for Gos/LP). The improved tumor inhibition may result from the tumortargeting effects and the sustained drug release of the liposomes. Particularly, Gos/cRGD-LP showed the most effective tumor suppression, with the tumor inhibition rate of $>74 \%$ on day 25 (Figure 9B), which was probably due to the enhanced tumor-targeting effect realized by the incorporation of cRGD into the liposome. To validate the effect of Gos/ cRGD-LP on tumor growth, a TUNEL assay was performed.

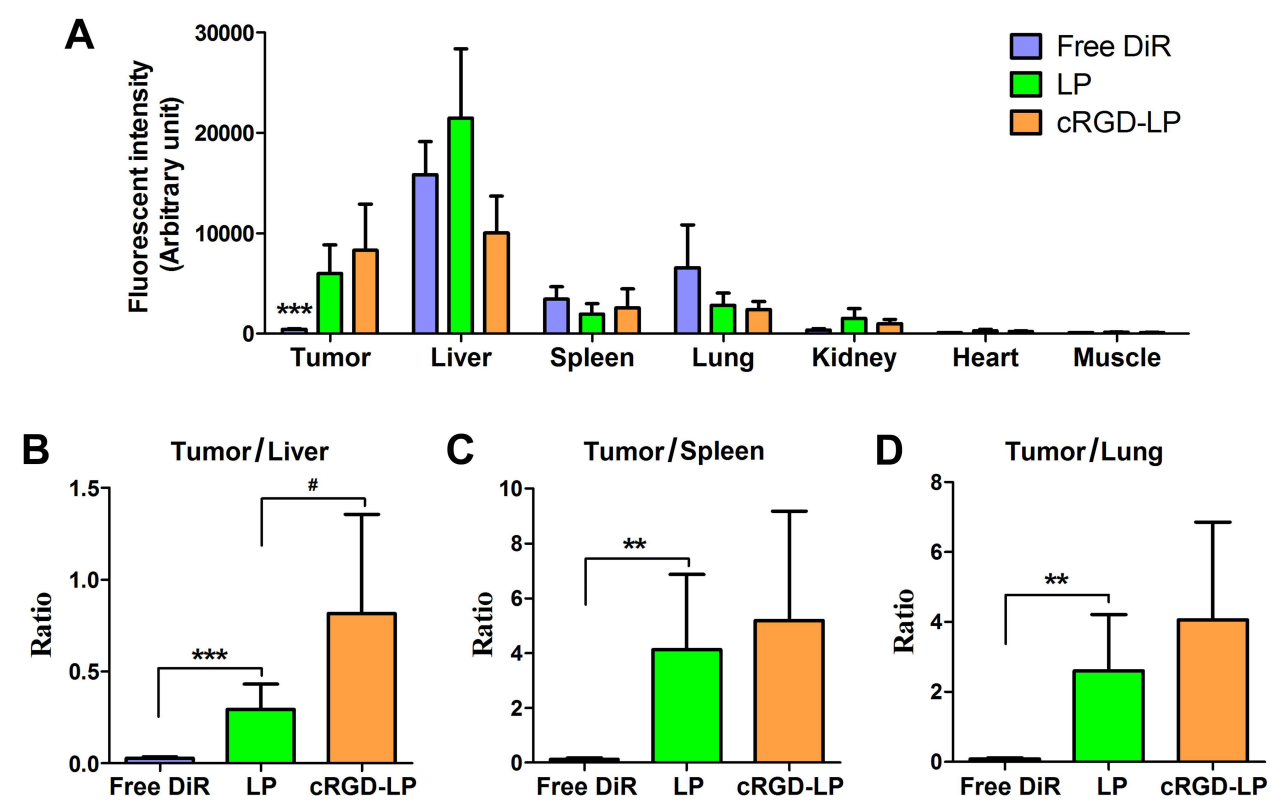

Figure 8 Quantitative analysis of ex vivo NIR fluorescence imaging. (A) The DiR fluorescence intensity of tumors and organs/tissues of the mice receiving DiR-loaded liposomes (cRGD-LP or LP) or free DiR. (B-D) Fluorescence intensity ratios of tumor to (B) liver, (C) spleen, and (D) lung. In figure $(\mathbf{A})$, data are shown as mean \pm SD $(n=$ 6 for tumor, and $n=3$ for organs/tissues). In figures (B-D), data are shown as mean $\pm S D(n=6)$. **p $<0.0$ I and ***p $<0.00$ I for LP as compared with free DiR; \#p $<0.05$ for CRGD-LP as compared with LP. 


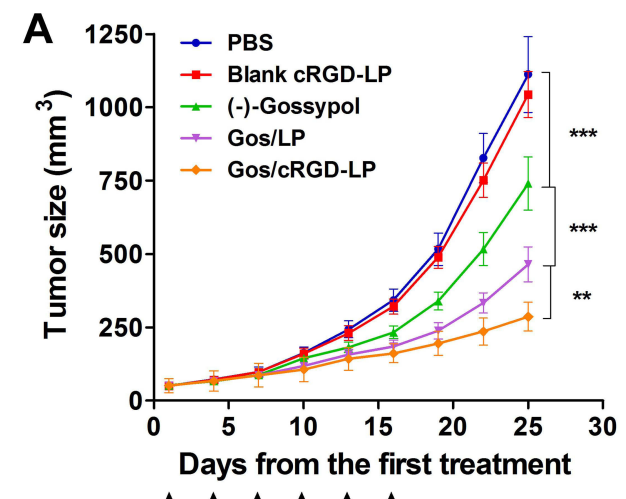

$\uparrow \uparrow \uparrow \uparrow \uparrow$
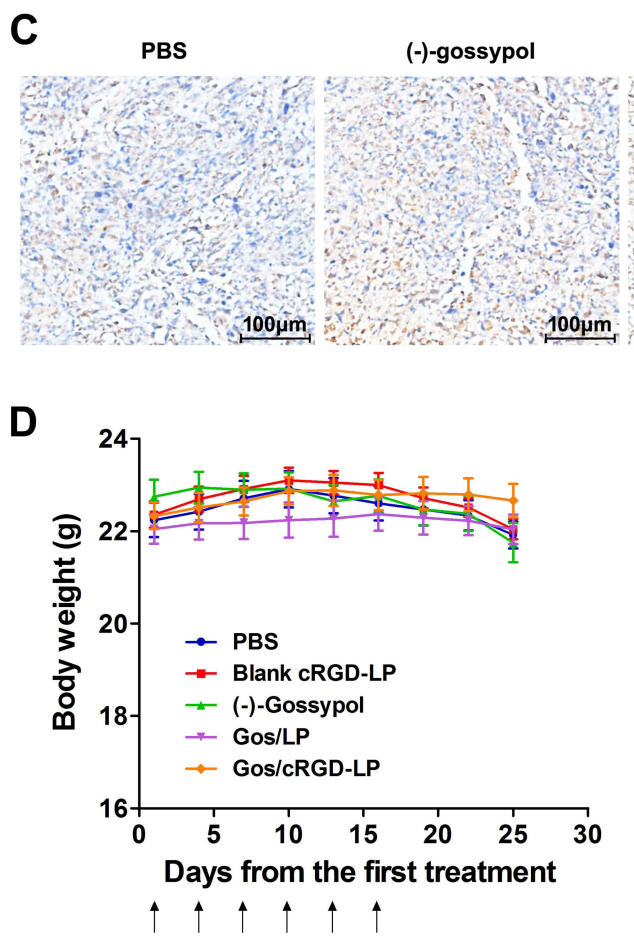

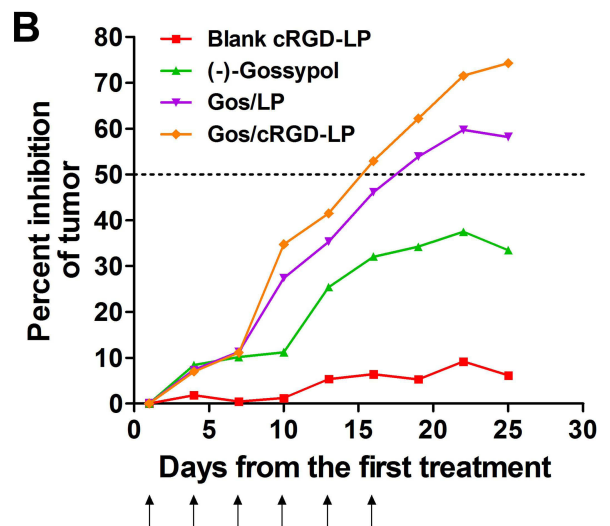

Gos/LP

Gos/cRGD-LP
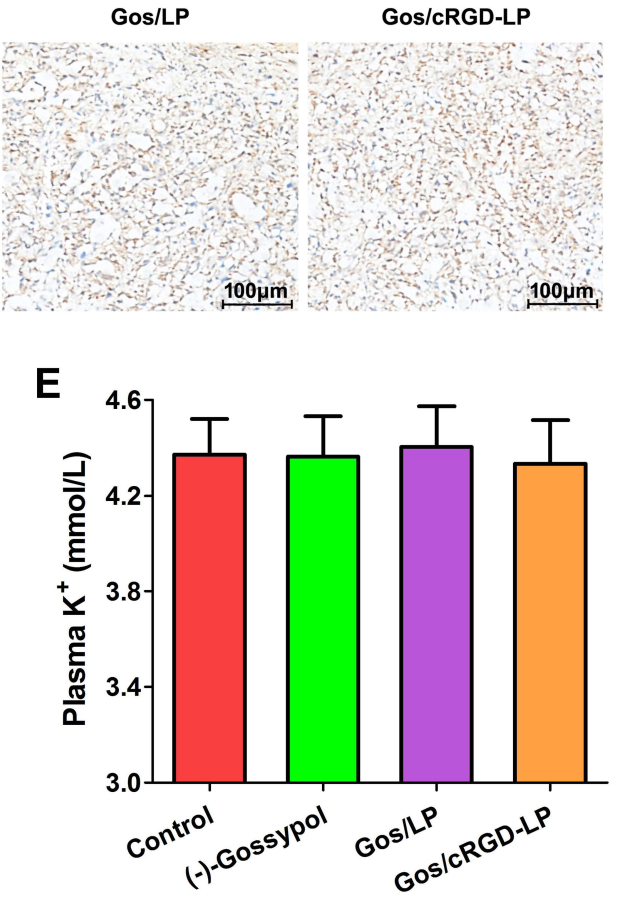

Figure 9 (A) In vivo tumor growth curves. PC-3 tumor-bearing nude mice were given PBS (i.v., blank control), blank cRGD-LP (i.v., vehicle control), free (-)-gossypol (P.O., $15 \mathrm{mg} / \mathrm{kg}$ ), Gos $/ \mathrm{LP}$ (i.v., $15 \mathrm{mg}$ (-)-gossypol $/ \mathrm{kg}$ ), or Gos/cRGD-LP (i.v., $15 \mathrm{mg}$ (-)-gossypol/kg). The arrows indicate the treatment time points. Data are shown as mean \pm SEM $(\mathrm{n}=8)$. On day 25, **P < 0.01 (Gos/cRGD-LP vs Gos/LP) and ***P < 0.001 [Gos/LP vs (-)-gossypol; and (-)-gossypol vs PBS]. (B) Changes in the tumor growth inhibition rate (IR) of each group as compared with the PBS group. The IR was over 50\% for Gos/cRGD-LP group after day I6, and for Gos/LP group after day I9. (C) Pathological section images of the tumor tissues from different groups. The blue stains indicated the normal tumor cells. The brown stains indicated the apoptotic body or apoptotic nucleus. (D) Changes in the body weight of each group. Data are shown as mean \pm SEM $(n=8)$. No significant change has been observed in the body weight of each group during the experiment. (E) The plasma potassium $(\mathrm{K}+)$ levels of mice after different treatments. Data are shown as mean \pm SD $(n=8)$. Nude mice were given free $(-)$-gossypol $(P . O$., $15 \mathrm{mg} / \mathrm{kg}$ ), Gos/LP (i.v., $15 \mathrm{mg}(-)$-gossypol/kg), or Gos/cRGD-LP (i.v., $15 \mathrm{mg}(-$ )-gossypol $/ \mathrm{kg}$ ), once every three days, for 6 times in total. The plasma K+ levels of the mice were tested on the day after the last treatment.

Figure 9C shows the pathological section images of the tumor tissues from different groups. The brown stains indicating the apoptotic body or apoptotic nucleus were not very abundant in the sample without the treatment of the drug (ie the PBS group). By contrast, considerable brown stains could be observed in the tumor tissue of the free (-)-gossypol group, indicating the apoptosis in the tumor cells induced by the drug. Nevertheless, compared with the free drug group even more extensive apoptosis could be observed in Gos/LP and Gos/
cRGD-LP groups, which demonstrated the enhanced apoptotic cell death realized by the tumor-targeted delivery of (-)gossypol. These findings were in accordance with the results of the fluorescence imaging and the drug efficacy studies.

The body weight loss of the mice may indicate the potential systemic toxicity and adverse effects of the drug. ${ }^{2,39}$ As shown in Figure 9D, within the dose schedule adopted for each group in this study, no significant change in the body weight was observed throughout the experiment. Among the five groups, 
the free (-)-gossypol group showed the largest body weight loss of $4.36 \%$ at the end of the experiment, as compared with the average body weight on day 1 , which may indicate slight systemic toxicity of the orally administered free drug. The orally administered (-)-gossypol could lead to gastrointestinalrelated side effects such as stomach discomfort, anorexia, and nausea/vomiting. ${ }^{10}$ Using injectable liposomal (-)-gossypol could minimize these gastrointestinal-related side effects of the drug. However, the biodistribution of (-)-gossypol is not specific to tumors, which may still lead to toxicity in the normal organs/tissues. Hypokalemia is a major adverse effect of gossypol (including its racemate and (-)-gossypol) that may happen after repeated/long-term treatments, which is related to the impacts of the drug on the renal tubules. ${ }^{2}$ Therefore, monitoring the plasma $\mathrm{K}^{+}$level is a need for the safety of repeated/ long-term use of gossypol. In this study, cRGD peptide with high affinity to $\alpha_{v} \beta_{3}$ integrin was incorporated into the liposome for enhanced tumor-targeted delivery of (-)-gossypol. Since integrin $\alpha_{v} \beta_{3}$ is overexpressed on both tumor vasculature and tumor cells, cRGD-LP could promote the accumulation of the drug in the tumor tissues rather than the normal tissues, which may effectively reduce the side effects of (-)-gossypol. Figure 9E shows the plasma $\mathrm{K}^{+}$levels of mice after different treatments. No significant change in the plasma $\mathrm{K}^{+}$levels was observed for the mice receiving free drug or the drug-loaded liposomes, as compared with the control mice without treatment. This result, together with the body weight changes, reflected the good safety of Gos/cRGD-LP and Gos/LP within the dose schedule adopted in this study.

In summary, Gos/cRGD-LP was a safe nano preparation for (-)-gossypol, with a good inhibitory effect on PC-3 tumors in vivo. The results from in vivo studies provided evidence supporting our hypothesis that the incorporation of cRGD into the (-)-gossypol-loaded liposome could enhance the tumor-targeting effect and consequently improve the antitumor efficacy of the drug in vivo. Nevertheless, it is noteworthy that the incorporation of integrin targeting ligands may block the potential integrin receptors. It has been reported that two $\alpha_{v} \beta_{3}$ antagonists, TDI-3761 and TDI-4161, block $\alpha_{\mathrm{v}} \beta_{3}$-mediated cell adhesion to ECM ligands. ${ }^{37}$ In this regard, although the fluorescence imaging has demonstrated the enhanced tumortargeting effect of the cRGD-LP, the incorporated cRGD may have competed with the potential integrin receptors involved in the integrin-mediated physiological and pathological processes, and the targeting ability as well as the therapeutic effect of the liposomal (-)-gossypol could be affected or regulated by these processes, which requires more detailed studies to verify. Indeed, there are still a lot of works to be done to further confirm the effects of cRGD on the tumor specificity and the drug efficacy of Gos/ cRGD-LP, and also to clarify the tumor-targeting mechanism of Gos/cRGD-LP. In the next studies, cell lines expressing high levels of integrin $\alpha_{v} \beta_{3}$ and those not expressing or expressing very low levels of integrin $\alpha_{\mathrm{v}} \beta_{3}$ will be adopted for in vitro and in vivo studies. The expression levels of integrin $\alpha_{v} \beta_{3}$ in the cells in vitro and the tumor xenografts and vasculature in animals will be analyzed, to make clear the effects of cRGD on the tumor specificity and the drug efficacy of Gos/cRGD-LP. Also, it is important to clarify the toxicity of Gos/cRGD-LP on healthy cells in vitro and healthy organs/tissues in animals, and the potential mechanisms. For this purpose, the apoptosis and autophagy of the cells will be evaluated, and the morphology of the cells and the histology of the tissues will also be studied.

\section{Conclusion}

A cRGD-decorated mixed liposome, Gos/cRGD-LP, was successfully developed for enhanced tumor-targeted delivery of the single-isomer drug (-)-gossypol. The cRGD-LP had a significantly enhanced tumor-targeting effect in vivo as compared with the non-cRGD-containing LP. The therapeutic efficacy of Gos/cRGD-LP was significantly better than that of Gos/LP. The increased in vivo tumor inhibition of Gos/cRGDLP as compared with Gos/LP was probably due to the enhanced tumor-targeting effect realized by the binding of cRGD with $\alpha_{v} \beta_{3}$ integrin on the tumor vascular endothelium and the tumor cells. These results indicated the potential of Gos/cRGD-LP that warrants further investigation for clinical applications of this single-isomer drug. Nevertheless, for a more comprehensive investigation of the tumor-targeting effect and antitumor efficacy of Gos/cRGD-LP and the mechanisms, cell lines expressing high levels of integrin $\alpha_{v} \beta_{3}$ and those not expressing or expressing very low levels of integrin $\alpha_{v} \beta_{3}$ will be adopted for in vitro and in vivo comparative studies.

\section{Acknowledgments}

This study was supported by Sichuan Science and Technology Program Project (2019YJ0489), the Joint Research Project of Southwest Medical University and the Affiliated Traditional Chinese Medicine Hospital of Southwest Medical University (2020XYLH-082), the Applied Basic Research Program of Southwest Medical University (2017-ZRQN-098), the Strategic Cooperation 
Project of the First People's Hospital of Neijiang and Southwest Medical University (2021NJXNYD07), and the Joint Research Fund of School of Chinese-Western Medicine Integration and the Affiliated Traditional Chinese Medicine Hospital of Southwest Medical University (to Houyin Shi).

\section{Disclosure}

The authors report no conflicts of interest in this work.

\section{References}

1. Benvenuto M, Mattera R, Sticca JI, et al. Effect of the BH3 mimetic polyphenol (-)-gossypol (AT-101) on the in vitro and in vivo growth of malignant mesothelioma. Front Pharmacol. 2018;9:1269. doi:10.3389/fphar.2018.01269

2. Liu H, Wang S, Shi H, et al. Gastric floating tablet improves the bioavailability and reduces the hypokalemia effect of gossypol in vivo. Saudi Pharm J. 2021;29(4):305-314. doi:10.1016/j. jsps.2021.03.001

3. Linder B, Kogel D. Autophagy in cancer cell death. Biology. 2019;8:82. doi:10.3390/biology8040082

4. Andersson T. Single-isomer drugs: true therapeutic advances. Clin Pharmacokinet. 2004;43(5):279-285. doi:10.2165/00003088200443050-00001

5. Zeng Y, Ma J, Xu L, et al. Natural product gossypol and its derivatives in precision cancer medicine. Curr Med Chem. 2019;26 (10):1849-1873. doi:10.2174/0929867324666170523123655

6. Masuelli L, Benvenuto M, Izzi V, et al. In vivo and in vitro inhibition of osteosarcoma growth by the pan Bcl-2 inhibitor AT-101. Invest New Drugs. 2020;38(3):675-689. doi:10.1007/s10637-019-00827-y

7. Kline MP, Rajkumar SV, Timm MM, et al. R-(-)-gossypol (AT-101) activates programmed cell death in multiple myeloma cells. Exp Hematol. 2008;36(5):568-576. doi:10.1016/j.exphem.2008.01.003

8. Meyer N, Zielke S, Michaelis JB, et al. AT 101 induces early mitochondrial dysfunction and HMOX1 (heme oxygenase 1) to trigger mitophagic cell death in glioma cells. Autophagy. 2018;14 (10):1693-1709. doi:10.1080/15548627.2018.1476812

9. Mei H, Lin Z, Wang Y, et al. Autophagy inhibition enhances pan-Bcl2 inhibitor AT-101-induced apoptosis in non-small cell lung cancer. Neoplasma. 2014;61(02):186-192. doi:10.4149/neo_2014_024

10. Baggstrom MQ, Qi Y, Koczywas M, et al. A Phase II study of AT-101 (gossypol) in chemotherapy-sensitive recurrent extensive-stage small cell lung cancer. $J$ Thorac Oncol. 2011;6 (10):1757-1760. doi:10.1097/JTO.0b013e31822e2941

11. Stein MN, Hussain M, Stadler WM, et al. A phase II study of AT-101 to overcome bcl-2-mediated resistance to androgen deprivation therapy in patients with newly diagnosed castration-sensitive metastatic prostate cancer. Clin Genitourin Cancer. 2016;14(1):22-27. doi:10.1016/j.clgc.2015.09.010

12. Zhang XQ, Huang XF, Mu SJ, et al. Inhibition of proliferation of prostate cancer cell line, PC-3, in vitro and in vivo using (-)-gossypol. Asian J Androl. 2010;12(3):390-399. doi:10.1038/aja.2009.87

13. Liu G, Kelly WK, Wilding G, et al. An open-label, multicenter, phase I/II study of single-agent AT-101 in men with castrate-resistant prostate cancer. Clin Cancer Res. 2009;15(9):3172-3176. doi:10.1158/ 1078-0432.CCR-08-2985

14. Xie H, Yin J, Shah MH, et al. A phase II study of the orally administered negative enantiomer of gossypol (AT-101), a BH3 mimetic, in patients with advanced adrenal cortical carcinoma. Invest New Drugs. 2019;37(4):755-762. doi:10.1007/s10637-01900797-1
15. Schelman WR, Mohammed TA, Traynor AM, et al. A Phase I study of AT-101 with cisplatin and etoposide in patients with advanced solid tumors with an expanded cohort in extensive-stage small cell lung cancer. Invest New Drugs. 2014;32(2):295-302. doi:10.1007/ s10637-013-9999-7

16. Swiecicki PL, Bellile E, Sacco AG, et al. A phase II trial of the BCL-2 homolog domain 3 mimetic AT-101 in combination with docetaxel for recurrent, locally advanced, or metastatic head and neck cancer. Invest New Drugs. 2016;34(4):481-489. doi:10.1007/ s10637-016-0364-5

17. Stein MN, Goodin S, Gounder M, et al. A phase I study of AT-101, a BH3 mimetic, in combination with paclitaxel and carboplatin in solid tumors. Invest New Drugs. 2020;38(3):855-865. doi:10.1007/ s10637-019-00807-2

18. Zerp SF, Stoter TR, Hoebers FJ, et al. Targeting anti-apoptotic Bcl-2 by AT-101 to increase radiation efficacy: data from in vitro and clinical pharmacokinetic studies in head and neck cancer. Radiat Oncol. 2015;10(1):158. doi:10.1186/s13014-015-0474-9

19. Heist RS, Fain J, Chinnasami B, et al. Phase I/II study of AT-101 with topotecan in relapsed and refractory small cell lung cancer. $J$ Thorac Oncol. 2010;5(10):1637-1643. doi:10.1097/JTO.0b013e3181e8f4dc

20. Flak DK, Adamski V, Nowaczyk G, et al. AT101-loaded cubosomes as an alternative for improved glioblastoma therapy. Int J Nanomed. 2020;15:7415-7431. doi:10.2147/IJN.S265061

21. Jin CL, Chen ML, Wang Y, et al. Preparation of novel (-)-gossypol nanoparticles and the effect on growth inhibition in human prostate cancer PC-3 cells in vitro. Exp Ther Med. 2015;9(3):675-678. doi:10.3892/etm.2015.2172

22. Tomoda K, Chiang HC, Kozak KR, et al. Injectable (-)-gossypol-loaded pluronic P85 micelles for cancer chemoradiotherapy. Int J Radiat Biol. 2017;93(4):402-406. doi:10.1080/09553002.2016.1257833

23. Li H, Piao L, Xu P, et al. Liposomes containing (-)-gossypol-enriched cottonseed oil suppress Bcl-2 and Bcl-xL expression in breast cancer cells. Pharm Res. 2011;28:3256-3264. doi:10.1007/s11095-011-0498-2

24. Li K, Liu H, Gao W, et al. Mulberry-like dual-drug complicated nanocarriers assembled with apogossypolone amphiphilic starch micelles and doxorubicin hyaluronic acid nanoparticles for tumor combination and targeted therapy. Biomaterials. 2015;39:131-144. doi:10.1016/j.biomaterials.2014.10.073

25. Liu H, Zhou X, Wang Y, et al. Mixed micelle as nanocarrier for etomidate: development, in vitro characterizations, and in vivo study on toxicity and anesthetic effects. J Drug Deliv Sci Technol. 2019;49:123-131. doi:10.1016/j.jddst.2018.10.038

26. Liu H, Li K, Lan L, et al. Double-layered hyaluronic acid/stearic acid-modified polyethyleneimine nanoparticles encapsulating (-)gossypol: a nanocarrier for chiral anticancer drugs. $J$ Mater Chem B. 2014;2(32):5238-5248. doi:10.1039/C4TB00539B

27. Danhier F, Feron O, Preat V. To exploit the tumor microenvironment: passive and active tumor targeting of nanocarriers for anti-cancer drug delivery. $J$ Control Release. 2010;148(2):135-146. doi:10.1016/j.jconrel.2010.08.027

28. Yang S, Wang D, Zhang X, et al. cRGD peptide-conjugated polyethylenimine-based lipid nanoparticle for intracellular delivery of siRNA in hepatocarcinoma therapy. Drug Deliv. 2021;28 (1):995-1006. doi:10.1080/10717544.2021.1928794

29. Fu S, Zhao Y, Sun J, et al. Integrin alphavbeta3-targeted liposomal drug delivery system for enhanced lung cancer therapy. Colloids Surf B. 2021;201:111623. doi:10.1016/j.colsurfb.2021.111623

30. Khabazian E, Vakhshiteh F, Norouzi P, et al. Cationic liposome decorated with cyclic RGD peptide for targeted delivery of anti-STAT3 siRNA to melanoma cancer cells. J Drug Target. 2021:1-34. doi:10.1080/1061186X.2021.1973481.

31. Ren Y, Yuan B, Hou S, et al. Delivery of RGD-modified liposome as a targeted colorectal carcinoma therapy and its autophagy mechanism. J Drug Target. 2021;29(8):863-874. doi:10.1080/ 1061186X.2021.1882469 
32. Liu H, Zhang Y, Hu M, et al. Film-injection as a dosage form for etomidate: enhancing the stability of nanomedicines using solid intermediate products. J Drug Deliv Sci Technol. 2020;56:101541. doi:10.1016/j.jddst.2020.101541

33. Liu H, Marquez RT, Wu X, et al. A non-intrusive evaluation method for tumor-targeting characteristics of nanomedicines based on in vivo near-infrared fluorescence imaging. J Mater Chem B. 2019;7 (31):4751-4757. doi:10.1039/c9tb00882a

34. Liu $\mathrm{H}$, Zhao $\mathrm{W}, \mathrm{Hu} \mathrm{Q}$, et al. Gastric floating sustained-release tablet for dihydromyricetin: development, characterization, and pharmacokinetics study. Saudi Pharm J. 2019;27(7):1000-1008. doi:10.1016/j. jsps.2019.08.002

35. Woolford L, Caraguel CGB, Taggart DA, et al. Serum biochemistry of free-ranging southern hairy-nosed wombats (lasiorhinus latifrons). J Zoo Wildl Med. 2020;50(4):937-946. doi:10.1638/2019-0001

36. Liu H, Gan C, Shi H, et al. Gastric floating pill enhances the bioavailability and drug efficacy of dihydromyricetin in vivo. J Drug DelivSci Technol. 2021;61:102279. doi:10.1016/j. jddst.2020.102279

37. Ludwig BS, Kessler H, Kossatz S, et al. RGD-binding integrins revisited: how recently discovered functions and novel synthetic ligands (re-)shape an ever-evolving field. Cancers. 2021;13(7):1711. doi:10.3390/cancers13071711

38. Rao TC, Ma VP, Blanchard A, et al. EGFR activation attenuates the mechanical threshold for integrin tension and focal adhesion formation. J Cell Sci. 2020;13:jcs238840. doi:10.1242/jcs.238840

39. Gao Y, Zhou Y, Zhao L, et al. Enhanced antitumor efficacy by cyclic RGDyK-conjugated and paclitaxel-loaded $\mathrm{pH}$-responsive polymeric micelles. Acta Biomater. 2015;23:127-135. doi:10.1016/j. actbio.2015.05.021

40. Almeida B, Nag OK, Rogers KE, et al. Recent progress in bioconjugation strategies for liposome-mediated drug delivery. Molecules. 2020;25(23):5672. doi:10.3390/molecules 25235672

41. Ruwizhi N, Aderibigbe BA. The efficacy of cholesterol-based carriers in drug delivery. Molecules. 2020;25(18):4330. doi:10.3390/ molecules 25184330

42. Mallick S, Thuy LT, Lee S, et al. Liposomes containing cholesterol and mitochondria-penetrating peptide (MPP) for targeted delivery of antimycin A to A549 cells. Colloids Surf B. 2018;161:356-364. doi:10.1016/j.colsurfb.2017.10.052
43. Danaei M, Dehghankhold M, Ataei S, et al. Impact of particle size and polydispersity index on the clinical applications of lipidic nanocarrier systems. Pharmaceutics. 2018;10(2):57. doi:10.3390/ pharmaceutics 10020057

44. Wessman P, Edwards K, Mahlin D. Structural effects caused by spray- and freeze-drying of liposomes and bilayer disks. J Pharm. Sci. 2010;99(4):2032-2048. doi:10.1002/jps.21972

45. Monnier Y, Farmer P, Bieler G, et al. CYR61 and alphaVbeta5 integrin cooperate to promote invasion and metastasis of tumors growing in preirradiated stroma. Cancer Res. 2008;68 (18):7323-7331. doi:10.1158/0008-5472.CAN-08-0841

46. Haubner R, Wester HJ, Burkhart F, et al. Glycosylated RGD-containing peptides: tracer for tumor targeting and angiogenesis imaging with improved biokinetics. J Nucl Med. 2001;42:326-336.

47. Remant BK, Thapa B, Xu P. pH and redox dual responsive nanoparticle for nuclear targeted drug delivery. Mol Pharmaceut. 2012;9 (9):2719-2729. doi:10.1021/mp300274g

48. Gu X, Wei Y, Fan Q, et al. cRGD-decorated biodegradable polytyrosine nanoparticles for robust encapsulation and targeted delivery of doxorubicin to colorectal cancer in vivo. $J$ Control Release. 2019;301:110-118. doi:10.1016/j.jconrel.2019.03.005

49. Fan X, Yuan Z, Shou C, et al. cRGD-conjugated $\mathrm{Fe}_{3} \mathrm{O}_{4} @$ PDA-DOX multifunctional nanocomposites for MRI and antitumor chemo-photothermal therapy. Int J Nanomed. 2019;14:9631-9645. doi: $10.2147 /$ IJN.S222797

50. Lu J, Zhao W, Huang Y, et al. Targeted delivery of doxorubicin by folic acid-decorated dual functional nanocarrier. Mol Pharmaceut. 2014;11(11):4164-4178. doi:10.1021/mp500389v

51. Lan L, Liu H, Smith AR, et al. Natural product derivative gossypolone inhibits musashi family of RNA-binding proteins. BMC Cancer. 2018;18(1):809. doi:10.1186/s12885-018-4704-z

52. Lu J, Zhao W, Liu H, et al. An improved D-alpha-tocopherol-based nanocarrier for targeted delivery of doxorubicin with reversal of multidrug resistance. J Control Release. 2014;196:272-286. doi:10.1016/j.jconrel.2014.10.016

53. Zhang X, Huang Y, Zhao W, et al. Targeted delivery of anticancer agents via a dual function nanocarrier with an interfacial drug-interactive motif. Biomacromolecules. 2014;15(11):4326-4335. doi:10.1021/bm501339j
International Journal of Nanomedicine

\section{Publish your work in this journal}

The International Journal of Nanomedicine is an international, peerreviewed journal focusing on the application of nanotechnology in diagnostics, therapeutics, and drug delivery systems throughout the biomedical field. This journal is indexed on PubMed Central, MedLine, CAS, SciSearch ${ }^{\mathbb{}}$, Current Contents ${ }^{\mathbb{R}} /$ Clinical Medicine, $^{-}$

\section{Dovepress}

Journal Citation Reports/Science Edition, EMBase, Scopus and the Elsevier Bibliographic databases. The manuscript management system is completely online and includes a very quick and fair peer-review system, which is all easy to use. Visit http://www.dovepress.com/ testimonials.php to read real quotes from published authors. 